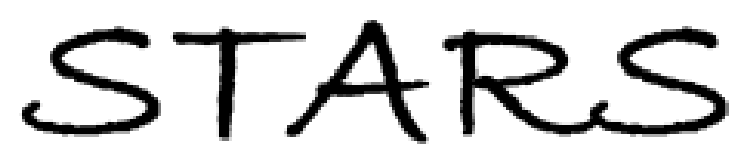

University of Central Florida

STARS

\title{
Shape Recovery Behavior of Carbon Nanopaper Shape Memory Polymer Composite
}

\author{
Veli Bugra Ozdemir \\ University of Central Florida
}

Part of the Mechanical Engineering Commons

Find similar works at: https://stars.library.ucf.edu/etd

University of Central Florida Libraries http://library.ucf.edu

This Masters Thesis (Open Access) is brought to you for free and open access by STARS. It has been accepted for inclusion in Electronic Theses and Dissertations by an authorized administrator of STARS. For more information, please contact STARS@ucf.edu.

\section{STARS Citation}

Ozdemir, Veli Bugra, "Shape Recovery Behavior of Carbon Nanopaper Shape Memory Polymer Composite" (2019). Electronic Theses and Dissertations. 6552.

https://stars.library.ucf.edu/etd/6552

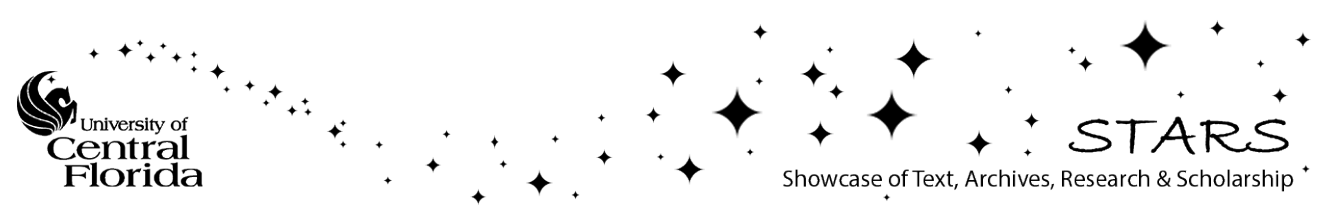


SHAPE RECOVERY BEHAVIOR OF CARBON NANOPAPER SHAPE MEMORY POLYMER COMPOSITE

by

\title{
VELI BUGRA OZDEMIR
}

B.S. Middle East Technical University, 2017

\begin{abstract}
A thesis submitted in partial fulfilment of the requirements for the degree of Master of Science in the Department of Mechanical and Aerospace Engineering in the College of Engineering and Computer Science at the University of Central Florida

Orlando, Florida
\end{abstract}

\section{Summer Term}

2019

Major Professor: Kawai Kwok 
(c) 2019 Veli Bugra Ozdemir 


\begin{abstract}
This thesis presents analytical, experimental and modeling studies of the shape recovery behavior of electrically activated Carbon Nanopaper (CNP) Shape Memory Polymer (SMP) composite. The composite structure studied consists of a CNP layer sandwiched by two SMP layers where the CNP layer acts as a flexible electrical heater when a voltage difference is applied. The behavior of CNP/SMP composite presents a coupled electrical - thermal - structural problem. The governing equations for the multiphysics behavior are derived. Derived parameters as a result of multiphysics analysis and effects of these parameters on the shape recovery behavior are investigated. The mechanical properties of the carbon nanopaper and viscoelastic properties of the shape memory polymer are characterized. A nonlinear, fully coupled electrical -thermal-structural finite element model is developed, and shape recovery experiments are carried out to validate multiphysics analysis and finite element model of the shape recovery of the CNP/SMP composite. Finite element model captures the general behavior of shape recovery, but overpredicts shape fixity and shape recovery rate.
\end{abstract}




\section{ACKNOWLEDGMENTS}

I would like to express my sincere gratitude to my advisor Dr. Kawai Kwok for his patience, guidance, support and ideas. I am truly grateful for having the opportunity to do research under your supervision and learn from your expertise.

I would like to thank my committee members Dr. Jihua Gou and Dr. Ranajay Ghosh for their appreciated comments and advice to improve my research.

I would like to thank my fellow lab mates, Alejandro Carrasco-Pena, Andrew Gomez-Delrio, Jayden Beyrooti, Milinda Yapa Hamillage and Wolfgang Klimm for their constant support, valuable suggestions and help over the years. 


\section{TABLE OF CONTENTS}

LIST OF FIGURES . . . . . . . . . . . . . . . . . . . . . viii

LIST OF TABLES . . . . . . . . . . . . . . . . . . . xii

CHAPTER 1: INTRODUCTION $\ldots \ldots \ldots \ldots \ldots \ldots \ldots \ldots \ldots$

Brief Background on Shape Memory Polymer and Shape Memory Polymer Composites . 1

Objectives of the Research . . . . . . . . . . . . . . . . 5

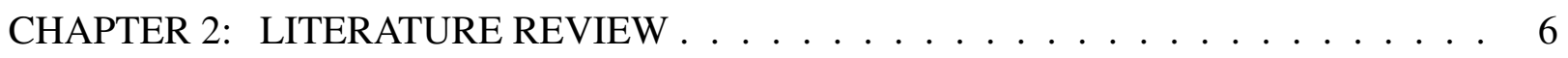

Shape Memory Polymers . . . . . . . . . . . . . . . . . . 6

Constitutive Models for Shape Memory Polymers _ . . . . . . . . . . . . . 7

Shape Memory Polymer Composites . . . . . . . . . . . . . . . . . . . 12

Carbon Nanopaper $\ldots \ldots \ldots \ldots$

CHAPTER 3: MULTIPHYSICS MODEL . . . . . . . . . . . . . . . . 17

Electro-thermal Analysis . . . . . . . . . . . . . . . . . 18

Thermo-mechanical Analysis . . . . . . . . . . . . . . 23

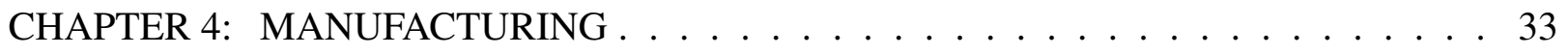




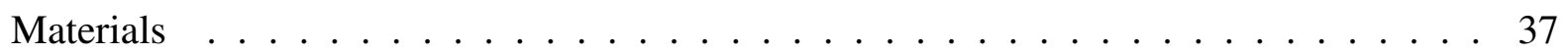

CHAPTER 5: CHARACTERIZATION OF CARBON NANOPAPER AND SHAPE MEMORY POLYMER . . . . . . . . . . . . . . . . . . . . . . . 39

Characterization of Shape Memory Polymer . . . . . . . . . . . . . . . . . . . . . 39

Carbon Nanopaper Characterization $\ldots \ldots \ldots$. . . . . . . . . . 48

CHAPTER 6: FINITE ELEMENT MODEL . . . . . . . . . . . . . . . . . . . . 52

CHAPTER 7: SHAPE RECOVERY EXPERIMENTS OF CNP/SMP COMPOSITE . . . . 56

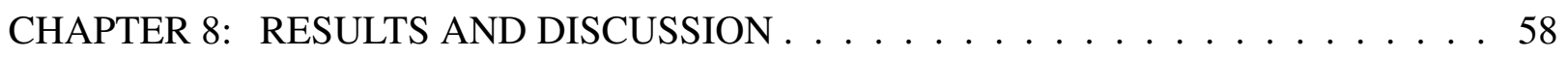

CHAPTER 9: CONCLUSIONS . . . . . . . . . . . . . . . . . . . . . 64

CHAPTER 10: FUTURE WORK . . . . . . . . . . . . . . . . . . . . . . 65

APPENDIX A: FINITE ELEMENT SIMULATIONS . . . . . . . . . . . . . . . . 66

Case: Sample thermal-electrical-structural simulation . . . . . . . . . . . . . 67

Case: Heat flux from one side . . . . . . . . . . . . . . . . . . . . . . . . . 69

Case: Bending simulation of CNP/SMP composite $\ldots \ldots \ldots \ldots 71$

APPENDIX B: SENSITIVITY ANALYSIS . . . . . . . . . . . . . . . 72 
APPENDIX C: SHAPE RECOVERY FORMULATION AND OPTIMIZATION FOR SHAPE CHANGE . . . . . . . . . . . . . . . . 77

APPENDIX D: STRESS RELAXATION EXPERIMENTS . . . . . . . . . . . . . . . . 82

LIST OF REFERENCES . . . . . . . . . . . . . . . . . . . . . . . . . . 87 


\section{LIST OF FIGURES}

Figure 1.1: Shape memory cycle of thermal responsive SMP . . . . . . . . . . . 1

Figure 1.2: Applications for fiber reinforced SMPs . . . . . . . . . . . . . . 3

Figure 1.3: Deployment of EMC Flexible Precision Reflector . . . . . . . . . . . . . 4

Figure 2.1: Micromechanics model for bi-phasic SMP model . . . . . . . . . . . 8

Figure 2.2: Change of resistivity with filler volume fraction $\ldots \ldots \ldots$

Figure 2.3: Resistivity change across cut-off distance . . . . . . . . . . . . 16

Figure 3.1: Concept model of CNP/SMP composite. . . . . . . . . . . . . . . . 17

Figure 3.2: Schematics of heat transfer between layers. . . . . . . . . . . . . 21

Figure 3.3: Heat transfer through shape memory polymer. . . . . . . . . . . 23

Figure 3.4: Programming and recovery of SMP . . . . . . . . . . . . . . . 24

Figure 3.5: Generalized Maxwell model _. . . . . . . . . . . . . . . . 25

Figure 3.6: Solution approach and relations between governing equations. . . . . . . . 32

Figure 4.1: Mold for SMP potting . . . . . . . . . . . . . . . . 33

Figure 4.2 : Degassing at $70^{\circ} \mathrm{C} \ldots \ldots \ldots \ldots \ldots$

Figure 4.3: Horizontal mold exploded view . . . . . . . . . . . . . . . 35 
Figure 4.4: Horizontal mold cut view . . . . . . . . . . . . . . . . 35

Figure 4.5: Vertical mold concept . . . . . . . . . . . . . . . 36

Figure 4.6: Final vertical mold concept . . . . . . . . . . . . . . . . 37

Figure 4.7: Front view of SMP/CNP composite $\ldots \ldots \ldots$. . . . . . . . . 37

Figure 4.8: SEM image of carbon nanopaper, magnification 406X . . . . . . . . . . 38

Figure 4.9: SEM image of carbon nanopaper, magnification $4900 \mathrm{X} \ldots \ldots$. . . . . . . . 38

Figure 5.1: Stress relaxation . . . . . . . . . . . . . . . . 40

Figure 5.2: Stress relaxation test setup _ . . . . . . . . . . . . . . . 41

Figure 5.3: Test specimen attached to grips . . . . . . . . . . . . . . . . . 42

Figure 5.4: Stress relaxation test results . . . . . . . . . . . . . . . 43

Figure 5.5: Master curve at $27^{\circ} \mathrm{C} \ldots \ldots \ldots \ldots$. . . . . . . . . . . . . 45

Figure 5.6: Shift factors . . . . . . . . . . . . . . . . . 46

Figure 5.7: One minute modulus, $E_{1}$ at different temperatures $\ldots \ldots \ldots$. . . . . . 48

Figure 5.8: Test setup for uniaxial tests of Carbon Nanopaper . . . . . . . . . . . . . . 49

Figure 5.9: Carbon Nanopaper specimen preparation . . . . . . . . . . . . . . . . 49

Figure 5.10Fracture patterns of Carbon Nanopaper . . . . . . . . . . . . . . . 50

Figure 5.11Carbon nanopaper tension tests . . . . . . . . . . . . . 50 
Figure 5.12 Hysteresis in cylic deformation . . . . . . . . . . . . . . . 51

Figure 6.1 : Meshed geometry of composite. . . . . . . . . . . . . . . 52

Figure 7.1: Shape recovery experiment setup. $\ldots \ldots \ldots \ldots 6$

Figure 8.1: Comparison of external force of FE model and experiment. . . . . . . . . 58

Figure 8.2: Comparison of shape recovery of FE model and experiment. . . . . . . . . 59

Figure 8.3: Temperature data from thermocouple readings and simulation. . . . . . . 60

Figure 8.4: Shape recovery with different actuation voltages. . . . . . . . . . . 61

Figure 8.5: Temperature field during heating $(\mathrm{t}=240 \mathrm{~s}) \ldots \ldots \ldots 2$

Figure 8.6: Stress field at stowage $(\mathrm{t}=403 \mathrm{~s}) . \ldots \ldots \ldots 2 \ldots \ldots \ldots$

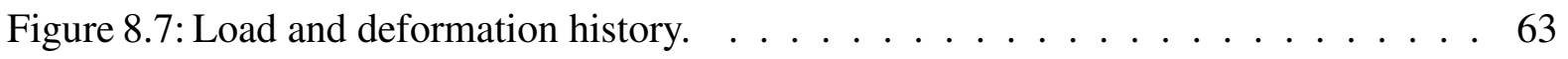

Figure 8.8: Thermo-mechanical cycle of composite. . . . . . . . . . . 63

Figure A.1:Temperature field during heating $\ldots \ldots \ldots \ldots$. . . . . . . 70

Figure A.2:Shape recovery of the composite in flexure $\ldots \ldots \ldots \ldots 71$

Figure B.1:Effect of interfacial thermal conductance on surface temperature $\ldots \ldots 73$

Figure B.2:Effect of interfacial thermal conductance on shape recovery . . . . . . . . 74

Figure B.3:Effect of deformation rate during programming $\ldots \ldots \ldots 75$ 
Figure B.4:Effect of hold duration during programming . . . . . . . . . . . . 75

Figure B.5:Effect of location of electrodes on potential field, located at half width . . . . 76

Figure B.6:Effect of location of electrodes on potential field, located at quarter width $\quad$. 76

Figure C.1:Shape recovery profile for different shift factors $\ldots \ldots \ldots$

Figure C.2:Temperature and shift factor history . . . . . . . . . . . . . . 79

Figure C.3:Shape recovery profile . . . . . . . . . . . . . . . . . 79

Figure C.4:Shape recovery profile defined by function $\varepsilon=1-\mathrm{t}^{2} / 10000 \ldots \ldots$

Figure C.5:Shift factor history for profile defined by $\varepsilon=1-\mathrm{t}^{2} / 10000 \ldots \ldots 1$

Figure D.1:Relaxation for different deformation levels . . . . . . . . . . . . . . 84

Figure D.2:Isochronus plot . . . . . . . . . . . . . . . . . . 84

Figure D.3:Sample strain field during stress relaxation test . . . . . . . . . . . 85

Figure D.4:Stress relaxation tests at different temperatures . . . . . . . . . . 85

Figure D.5:Shifted stress relaxation tests . . . . . . . . . . . . . 86

Figure D.6:Sorted master curve . . . . . . . . . . . . . . . . 86 


\section{LIST OF TABLES}

Table 2.1: Shape memory polymer classification . . . . . . . . . . . 6

Table 5.1: Prony series coefficients . . . . . . . . . . . . . . 46

Table 5.2: WLF Constants . . . . . . . . . . . . . . . . . 47

Table 6.1: Material parameters for FE model $\ldots \ldots \ldots$

Table A.1: Sample model parameters . . . . . . . . . . . . . . . . . . 67

Table A.2: Comparison of analytical solution and simulation . . . . . . . . . . . 69 


\section{CHAPTER 1: INTRODUCTION}

\section{Brief Background on Shape Memory Polymer and Shape Memory Polymer Composites}

Shape memory polymers (SMPs) are a type of smart material, which can change their shape from a deformed configuration to their original configuration under the application of stimuli such as heat, $\mathrm{pH}[1,2]$, moisture $[3,4]$, chemical [5], light $[6,7,8]$, and magnetism $[9,10]$, etc. After their discovery in 1980s, literature on SMPs mostly focuses on thermally activated polymers. SMPs have distinct advantages over the use of shape memory alloys (SMAs) such as low density, a range of activation temperatures, being easy to process [11], and recoverable strains up to $400 \%$ [12]. Due to their mentioned advantages, they possess high potential for applicatons ranging from space deployable structures to biomedical devices $[13,14]$.

Thermal responsive SMPs are the most commonly used type and the typical thermo-mechanical cycle for SMP is given in Figure 1.1 [15]. The ability to retain their programmed shapes in the absence of external stimuli makes SMP attractive in applications where there is an ever increasing need for smaller stowed volume and precise deployment behavior.

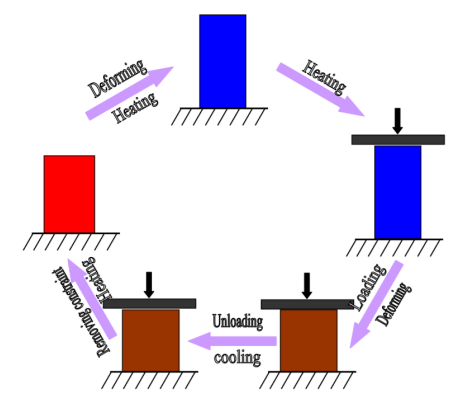

Figure 1.1: Shape memory cycle of thermal responsive SMP 
Deployment of heavy space structures may suffer from large shocks during deployment [15]. Also, advances in solar sails and solar panels pose a challenge to design lighter deployable structures with lower stowed volume [16]. The need for efficient usage of volume makes it difficult to employ traditional deployable structures [15]. Precise and full deployment of the stowed structure are other vital characteristics of favored deployable structures.

Over the years, shape memory polymers have drawn great attention due to their ability to meet these requirements. SMPs can preserve their shape in programmed configuration and can recover from high deformations, which makes them a suitable candidate to be used in ultralight deployable space structures. Although they are too compliant to be used as a structural member alone, many researchers and companies have focused on SMP composites with a reinforcing phase in the last decades. SMPs and their reinforced composites (SMPCs) are commonly used in aerospace applications as hinges [17] in solar arrays [18, 19], reflector antennas [15, 20], and morphing structures [21]. Figure 1.2 and Figure 1.3 show examples of SMPCs in deployable space applications. In the case of deployable hinges (Figure 1.2), a folded composite hinge can be deployed by activating SMP in the hinge region. Elastic Memory Composite (EMC) from Composite Technology Development (CTD), Inc. has merged high stiffness of fiber reinforced composites with shape recovery properties of SMPs and several improvements have been suggested to improve performance of the developed composite design over the years [22, 23]. Additionally, employment of SMPCs in deployable booms bypasses complex structural designs needed for deployment mechanisms. Different SMPC boom designs have been studied in literature ranging from coilable booms to foldable booms [19]. 


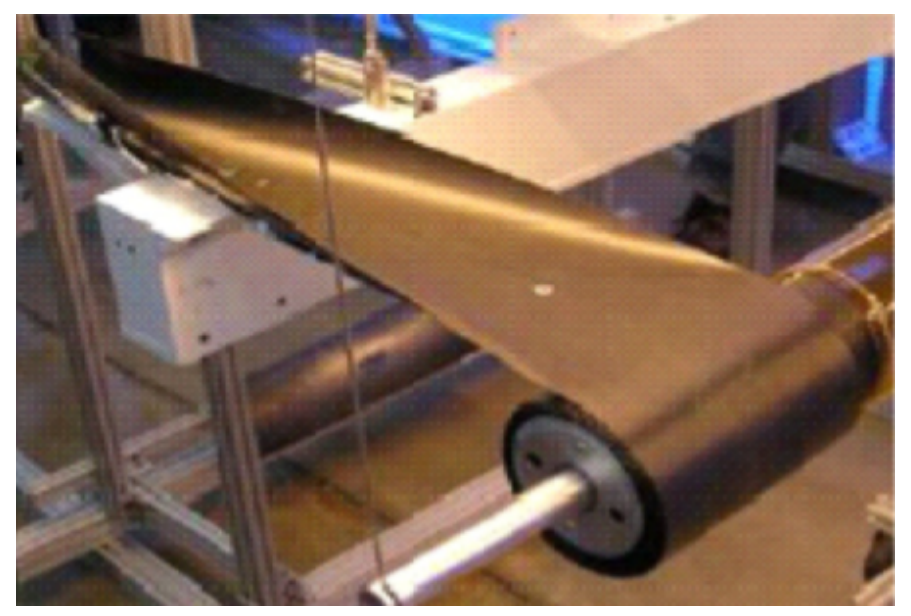

(a) Partially deployed EMC boom

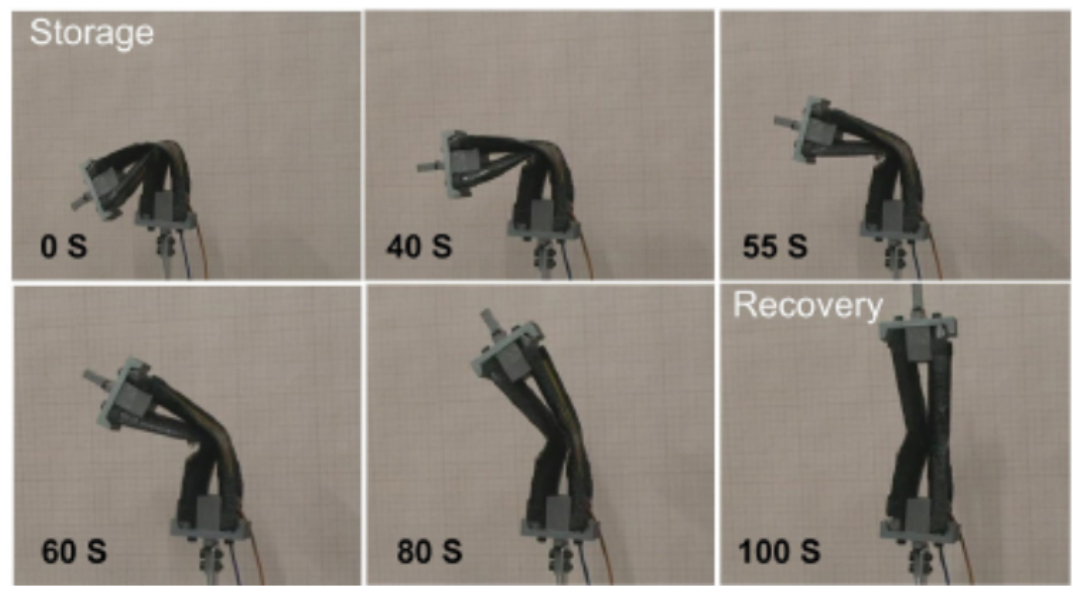

(b) Deployable hinge

Figure 1.2: Applications for fiber reinforced SMPs 


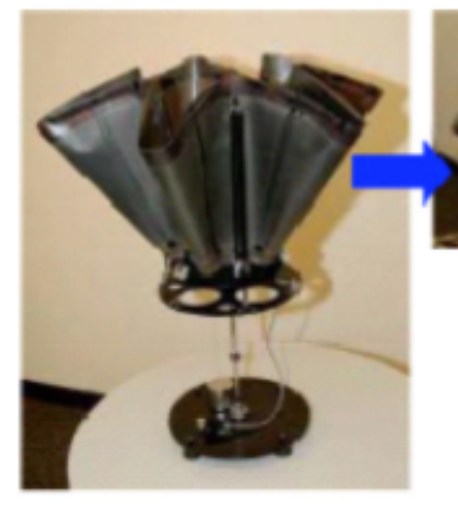

Fully Packaged

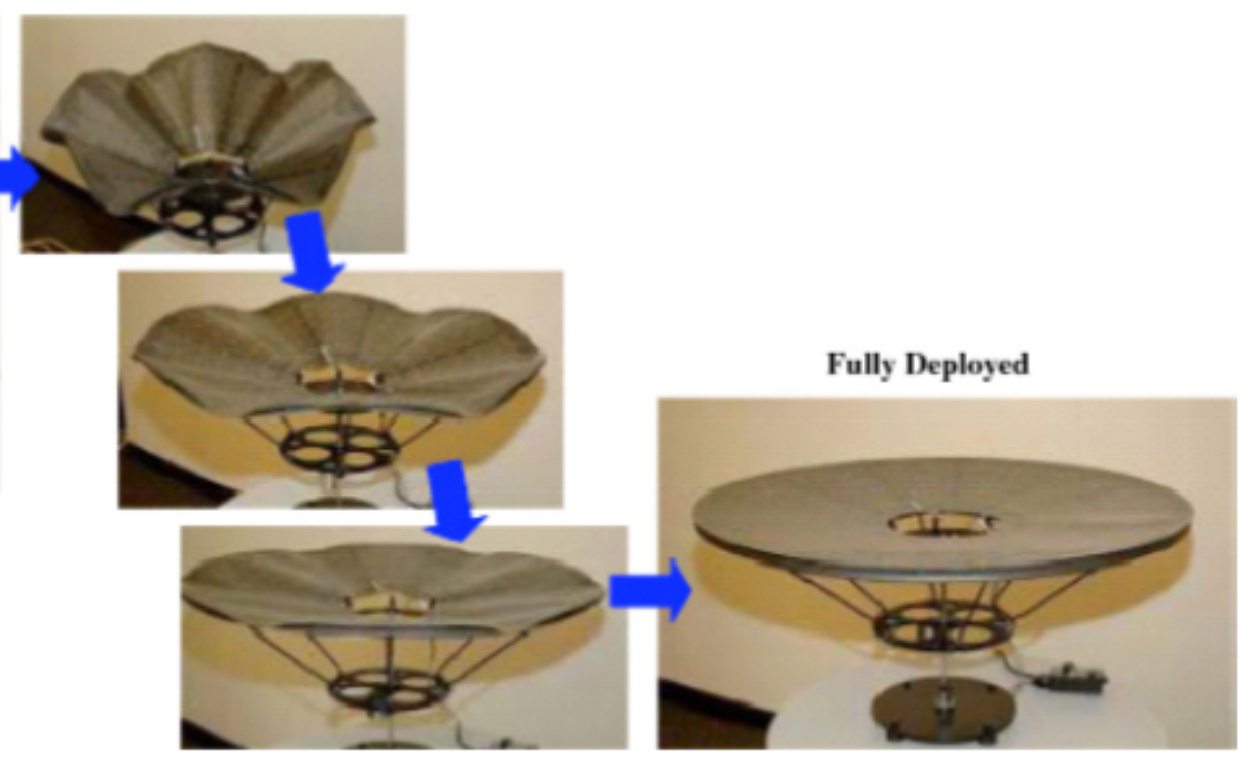

Figure 1.3: Deployment of EMC Flexible Precision Reflector

Different means of application of heat have been studied by various research groups: the convective heating, Joule heating [24, 25] and radiation on black-inked surface [26]. Among these methods of thermal actuation, Joule heating is the most versatile in terms of control and effectiveness which results in higher shape recovery rate. Joule heating is achieved by passing a current in electrically conductive layer, which in return heats up the SMP. Different metals can be used as conductive layer, however they display plasticity at low strain levels. Recent developments in carbon nanofiber technology made them suitable to be used as electrical heater. Carbon nanopapers (CNPs) are electrically conductive papers made of carbon nanofibers or carbon nanotubes and carbon nanopapers display higher strain limits compared to metals. CNPs have low density and high flexibility which make them advantageous in deployable space structures applications. 
Also, carbon nanopapers posses piezoresistive properties and can be used as a strain sensor. With the strain sensing capabilities of carbon nanopapers [27], they can be employed in CNP/SMP composite as both sensor and actuator component to activate different regions of the structure in timely manner to create complex geometries. In addition, they can be utilized in closed loop control for actively controlling shape recovery behavior of CNP/SMP composite in the future.

\section{Objectives of the Research}

In this work, the focus lies on the shape recovery behavior of thermally activated CNP/SMP composite via Joule heating. Although different material models regarding shape memory behavior have been developed and different heating methods have been investigated, previous research involves numerical simulations and experimental results but there is a lack of detailed multiphysics discussion in the composite behavior. In this thesis, a solution approach to the multiphysics problem is presented which aims to elucidate the physics of the problem and important parameters affecting the composite behavior. A fully coupled electrical-thermal-structural finite element model is proposed and experimental investigation are carried out to evaluate the performance of the model. 


\title{
CHAPTER 2: LITERATURE REVIEW
}

\author{
Shape Memory Polymers
}

Shape memory polymers can be classified in different ways, i.e. the method of activation, chemical and physical compositions etc. Liu et al.[28] proposes to divide SMPs into four classes (class IIV). Class I is covalently crosslinked glassy thermoset networks. Class I SMPs show rubbery elasticity for temperatures above $T_{g}$, and they show high shape recovery due to their covalent crosslinking. Class II SMPs are covalently crosslinked semi-crystalline networks, they can be tailored to display improved shape recovery compared to class I type. Class III and class IV SMPs are physically crosslinked and they are glassy and semi-crystalline, respectively. Latter classes show improved reshapebility. Semi-crystalline SMPs (classes II and IV) show the effect of the melting temperature of the semi-crystalline regions $T_{m}$ in shape recovery. Table 2.1 summarizes the properties of different classes of shape memory polymer.

Table 2.1: Shape memory polymer classification

\begin{tabular}{|c|c|c|c|c|}
\hline$\cdot$ & Class I & Class II & Class III & Class IV \\
\hline Crosslinking & Covalent & Covalent & Physical & Physical \\
\hline Reshapebility & Low & Low & High & High \\
\hline Crystalline regions & - & + & - & + \\
\hline Shape recovery ratio & $95-100 \%$ & $30-100 \%$ & $75-100 \%$ & $56-100 \%$ \\
\hline
\end{tabular}

SMPs can also be categorized as one-way or two-way SMPs. Although literature in SMAs have reported two-way shape memory alloys, the literature in two-way SMPs has been scarce.

In the literature, the performance of SMPs is commonly evaluated and compared using shape fixity and shape recovery ratios. Shape fixity is a measure of ability to retain programmed shape, and is 
given by Eq. (2.1)

$$
R_{f}=\frac{\varepsilon_{r}}{\varepsilon_{0}}
$$

where $\varepsilon_{r}$ is the strain after removing the deformation constraint, and $\varepsilon_{0}$ is the programming strain. Ideally, shape fixity should be close to 1 , suggesting the programmed shape can be maintained after removal of the constraint. The shape recovery ratio is used to evaluate the performance of the SMP during the activation cycle. It can be defined as,

$$
R_{r}(t)=1-\frac{\varepsilon(t)}{\varepsilon_{0}}
$$

where $\varepsilon(t)$ is the strain at a given time, and final value of $\varepsilon(t)$ can be used to evaluate the shape recovery performance at the end of the thermo-mechanical cycle. For fully recovered shape, shape recovery ratio should be 1 (ie. $\varepsilon(t)$ should be 0 ). These parameters are used to compare the performance of SMPs after undergoing repeated thermo-mechanical cycles. Schmidt et al. [29] has shown experimentally that, as the number of cycles increases, the shape recovery ratio (comparing with the initial shape) decreases due to residual strains from previous cycles, but the marginal effect of residual strain over a cycle becomes less for higher cycles.

\section{Constitutive Models for Shape Memory Polymers}

Constitutive modeling for shape memory polymers have been investigated widely. In literature, two main approaches have been used to explain the shape memory effect of SMPs[12, 30, 31]. The first approach utilizes the phase transition modeling approach while the second approach uses thermoviscoelastic material models. Nguyen [30] suggests phase transition modeling can be used 
in different classes of SMPs, such as crystallizable and amorphous polymers. Leng et al. [12] states that the capability to attain large deformations proves difficult to obtain a mechanical constitutive behavior.

Shape memory polymer models can also be divided into small strain models and finite deformation models. Finite deformation models are commonly an extension of the validated small strain models.

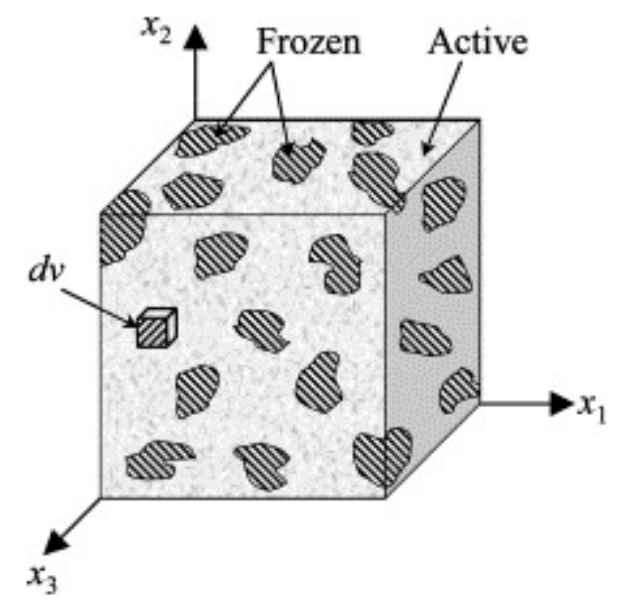

Figure 2.1: Micromechanics model for bi-phasic SMP model

The phase transition approach treats SMPs as a material consisting of different phases, mainly rubbery and glassy phases. The material response is determined by the volume fraction of these phases, and above $T_{g}$, the material response is determined by the rubbery phase [30]. Liu et al. [32] treated SMP as bi-phasic system (frozen and active phases in Figure 2.1) [32] and their volume 
fractions are given by,

$$
\phi_{f}=\frac{V_{f}}{V} \quad \phi_{a}=\frac{V_{a}}{V}
$$

where $\mathrm{V}$ is total volume, $V_{f}$ and $V_{a}$ are frozen and active volumes, respectively. With the assumption of slow strain rate and slow heating/cooling rate, the volume fractions will be a function of temperature only. With the assumption of stresses being equal in the phases, they defined total strain to be the summation of strains in two phases with rule of mixture, i.e.

$$
\varepsilon=\phi_{f} \varepsilon_{f}+\left(1-\phi_{f}\right) \varepsilon_{a}
$$

and $\varepsilon_{f}$ consists of three parts (frozen entropic strain, internal energetic strain and thermal strain) while $\varepsilon_{a}$ is divided into entropic strains and thermal strains. Finally writing generalized Hooke's law for active and frozen phases and combining them to yield the mechanical strain after some manipulations, the constitutive relation is presented by

$$
\sigma=\left(\phi_{f} S_{i}+\left(1-\phi_{f}\right) S_{e}\right)^{-1}:\left(\varepsilon-\varepsilon_{s}-\varepsilon_{T}\right)
$$

where $S_{e}$ and $S_{i}$ are fourth order tensor for entropic deformation and internal energetic deformation, respectively. As a result, only two state variable remains: $\varepsilon_{s}$ (stored strain) and $\phi_{f}$. The work of Liu et al. has been improved over the years by other researchers and the shape recovery mechanism has become more clear by the work of Chen et al. [33] who extended the model to large deformations using Neo-Hookean material model as a special case.

The thermoviscoelastic approach takes into account the time-dependent properties. This approach 
uses the free volume concept and chain mobility to explain shape fixity and recovery of SMPs. At low temperatures, the free volume for chains to move easily is low and the viscosity of the medium is high, however at higher temperatures, chains can move more freely in less viscous medium. This approach is commonly used in conjcution with relaxation or retardation times and time-temperature superposition principle to account for the effect of the temperature. Attempts to explain the shape recovery effect via viscoelastic approach resulted in numerous models. Lin et al.[34, 35], modeled SMP with two Maxwell models in parallel for polyurethanes with the Williams-Landel-Ferry equation for accelerated response of material (via shift factors). An analytical solution is presented from two Maxwell elements. The model presented in that paper together with the paper by Qi et al. [36] are adopted for this thesis. Other viscoelastic models in the literature include the works of Tobushi et al. [37], where linear and nonlinear constitutive models are presented. The linear constitutive model [38] is given by,

$$
\dot{\varepsilon}=\frac{\dot{\sigma}}{E}+\frac{\sigma}{\mu}-\frac{\varepsilon-\varepsilon_{s}}{\lambda}+\alpha \dot{T}
$$

where $\lambda, \mu, \alpha$ are retardation time, viscosity and the coefficient of thermal expansion, respectively. Here, $\varepsilon_{s}$ is defined as irrecoverable strain. The nonlinear constitutive model is constructed by adding nonlinear terms expressed by power of stress,i.e.

$$
\dot{\varepsilon}=\frac{\dot{\sigma}}{E}+m\left(\frac{\sigma-\sigma_{y}}{k}\right)^{m-1} \frac{\dot{\sigma}}{k}+\frac{\sigma}{\mu}+\frac{1}{b}\left(\frac{\sigma}{\sigma_{c}}-1\right)^{n}-\frac{\varepsilon-\varepsilon_{s}}{\lambda}+\alpha T
$$

where $\sigma_{y}$ is the yield stress, whereas $\sigma_{c}$ is the creep limit.

Lai and Baker [39] developed a 3-D Schapery representation for nonlinear viscoelasticity, noting that the use of simple classical constituitive models may not be adequate in practical applications. 
The developed constituitive model is converted to incremental form to study material response. Their main assumption is that the deviatoric and dilational behavior of the material are uncoupled. Time-temperature superposition principle is implemented while considering the effect of physical ageing of polymers and deformation dependence of shift factors. They have also stated that the choice of the time step in numerical integration has no effect on accuracy, however it can cause convergence issues when significant changes are involved during this time step.

By tailoring $T_{g}$ via polymer chemistry (monomer and/or comonomer type and ratio, crosslink density) and processing, desired effects can be observed for given operational temperature range [40]. Over the years, high dependence of performance of SMPs in different applications forced researchers to develop SMPs with specified $T_{g}$ (varying from $35^{\circ} \mathrm{C}$ to $113^{\circ} \mathrm{C}$ ) [12].

Baghani et al. [31] developed a finite deformation constituitive model based on logarithmic strain. In that paper, the material is modeled with rubbery and glassy phases with varying volume fraction depending on the temperature. For each phase, the deformation gradient is decomposed into glassy and rubbery parts. Naghdabadi et al. [41] criticizes the use of spring and dashpot elements in SMP modeling and states that the response of these models only agree qualitatively with experiments.

Castro et al. [42] discusses the effect of programming and activation conditions on the shape recovery rate, by considering isothermal tests and shape memory cycle tests. Faster shape recovery is achieved under isothermal conditions compared to shape memory cycle conditions with the same recovery temperature. Faster recovery can be achieved at lower programming temperature and higher activation temperature. These results can be explained by the high elastic response of the material under isothermal conditions and the effect of activation temperature. Castro et al. [42] have also noted that, if the activation temperature is lower than the programming temperature, shape recovery occurs at a much slower rate. 


\section{Shape Memory Polymer Composites}

SMPs are highly compliant materials and show lower mechanical strength compared to other materials, therefore, over the last two decades, many researchers and companies focused on the use of shape memory polymers in fiber reinforced composites $[12,43]$. Studies have been conducted where shape memory polymers are employed as matrix material. In the literature, the fiber reinforced shape memory polymer matrix composites are commonly referred as Elastic Memory Composites (EMCs) or SMPCs. Use of EMCs results in higher packaging and lower stowed volumes compared to traditional composite structures. Comparing with the traditional polymer matrix composites, employing SMP as matrix material was found to improve nominal bending strains without causing failure of the fibers. During packaging of the structure, high temperature causes SMP to lose its stiffness drastically (on the order of several hundreds), which allows fibers to form continuous microbuckles on the compression side [44]. Instead of using a low modulus material as matrix [45, 46, 47], using SMP to facilitate its variable stiffness property can help to achieve high curvatures during packaging and higher stiffness in the programmed configuration.

Bergman et al. [48] developed a finite element model for SMPC beams to study the effect of dynamic deployment for unidirectional fiber composite with different orientation angles. Bergman et al. have shown that the shape fixity of SMPCs are greatly affected by volume fraction of fibers and orientation angle. This result is intuitive due to the high modulus of fibers compared to SMP, with $10 \%$ fiber volume fraction, after unloading, more than $90 \%$ of the initial shape is recovered. For $90^{\circ}$ orientation of fibers, shape fixity can be approximated with only SMP response. When designing deployable space structure, fiber orientation angles and stowed shape requirements must be taken into account. If high shape fixity is required under the load-free state, the composite should be programmed accordingly to avoid high stored energy in the fibers. Meng et al. [49] state that the reason for the lack of updated research in SMPCs is due to the fact that shape memory 
effect becomes negligible in reinforcement direction.

Apart from the reinforcing aspect, the use of carbon nanofibers (CNFs) and carbon nanotubes (CNTs) can be used with SMP matrix due to their high conductivity. Several researchers have focused on manufacturing aspect of implementing CNF and CNTs into SMP matrix uniformly and several manufacturing methods have been developed.

Electrically actuated SMP composites have been under investigation extensively [50, 51, 52, 53, 54]. The conductive materials in SMP composites acts as an internal heating mechanisms under the application of current to the conductive layer. Different conductive layers have been used such as carbon black, short carbon fibers [55, 56], carbon nanotubes, metals [57], or combination of them to improve shape recovery performance [58].

\section{Carbon Nanopaper}

The literature regarding the piezoresistivity of carbon nanopapers is vast. CNTs possess high thermal and electrical conductivities [59], as well as high stiffness and strength.

Carbon nanotubes (CNTs) filled polymer composites have been investigated widely for their multifunctional behavior such as piezoresistive sensing, flexibility and high conductivity. Typical sensing applications can include wearable electronics and medical health monitoring [60]. In the literature, the main focus is on developing a model to capture piezoresistive properties of these materials. Several numerical studies have been published to quantify the effect of different parameters such as the aspect ratio of fillers [60, 61], volume fraction of fillers, alignment of CNTs [59], etc. Wang et al. [60] state that the average junction gap variation can be used as a quantitative parameter to describe the resistive behavior of a CNT network upon strain. 
The work by Zare et al. [62] focused on the modeling of the tunneling conductivity of polymer/CNT nanocomposites considering the effects of tunneling distance and CNT waviness. They have considered an extended CNT approach where tunneling space (interphase) is included in the modeled CNT network. Different configurations for tunneling distance definitions have been used from Takeda's work. Clearly, the interphase thickness will change the conductivity, critical volume fraction, percolation threshold etc. Later, experimental data was used with the suggested model to conclude the effects of each parameters. Similar to Zare et al., Wang and Zhao [63] focused on developing a simple analytical expression for conductivity and piezoresistive behavior.

One of the most important aspects related to conductive network of CNTs is the percolation threshold, which is the critical volume fraction of CNTs where the first conductive path forms. Conductivity can be thought as negligible for the volume fractions below the percolation threshold. Mertiny et al. [64] developed a 3-D Monte Carlo model for nano-disks to study the onset of percolation. Figure 2.2 [64] shows change in resisitivity with the volume fraction of the fillers. Main contribution to conductance arises from conductive path formation in the composite. The probability of forming a conductive path highly depends on the volume fraction of CNT fillers. In order to conduct a current, CNTs does not need to form a hard contact with each other. If CNTs are close enough, electrons can transport between CNTs, a phenomenon called the tunneling effect. In this case, the overall resistance of the composite will consist of intrinsic resistance of CNTs as well as the resistance due to tunneling. In the literature, for electrical resistivity via tunneling effect is widely accepted as Eq. 2.8 [64];

$$
R_{\text {tunneling }}=\frac{h^{2} d}{A e^{2} \sqrt{2 m \lambda}} \exp \left(\frac{4 d \pi \sqrt{2 m \lambda}}{h}\right)
$$

where $\mathrm{m}$ is the mass of an electron, $\mathrm{h}$ is the Planck's constant, $\lambda$ is the barrier height of the polymer, e is the quantum of electricity, $\mathrm{d}$ is the tunneling distance and A is the cross-sectional area of tunnel. 
The tunneling distance is an important parameters and the value of d greatly affects the tunneling condition. If $\mathrm{d}$ is large enough, electrons cannot jump between CNTs and conductivity decreases drastically. The critical value of $d$ is called cut-off distance Figure 2.3 [61]. Following work from Mertiny et al. [61] improved model for volume fractions larger than percolation threshold.

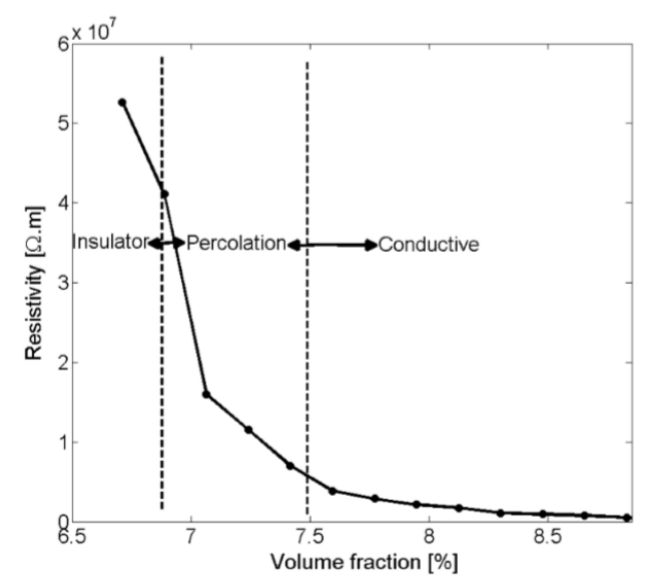

Figure 2.2: Change of resistivity with filler volume fraction

Carbon nanotube based composites are also investigated for their electromagnetic shielding effects in spacecraft applications together with their high thermal and electrical conductivity [65]. This application can overcome charge accumulation in spacecraft surfaces via an electrical discharge mechanism.

Regarding manufacturing, different methods have been studied to improve the properties of CNT/polymer composites, main focus being the alignment of CNTs in polymer substrate [66]. The model developed by Takeda et al. [59] suggests that straightening of sufficiently long CNTs during manufacturing can increase composite conductivity. 


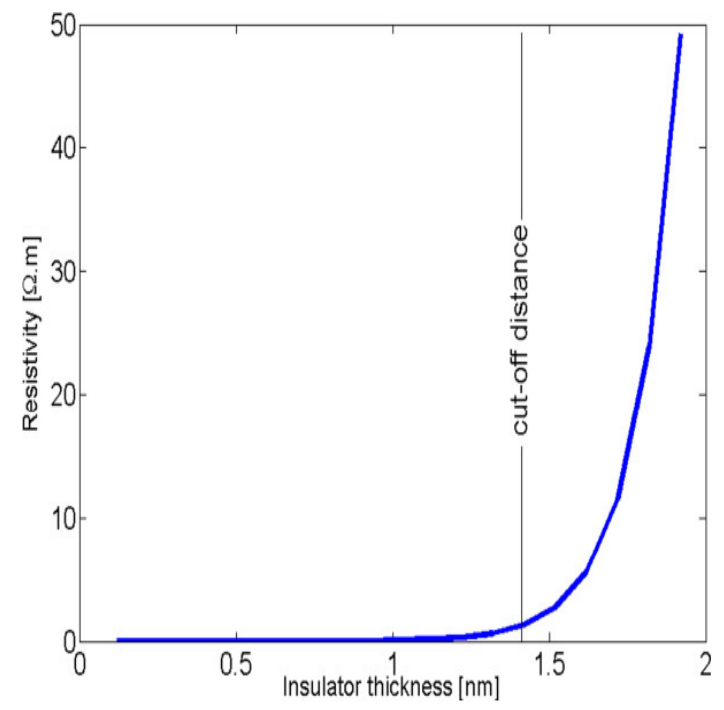

Figure 2.3: Resistivity change across cut-off distance 


\section{CHAPTER 3: MULTIPHYSICS MODEL}

The particular CNP/SMP composite under study is shown in Figure 3.1. The composite is a sandwich structure which consists of three layers, one layer of CNP and two layers of SMP. The power source is connected to the ends of the CNP layer, giving a voltage difference of $\mathrm{V}(\mathrm{t})$. Due to current passing through this layer, heat will be dissipated in the CNP via Joule heating. Some fraction of this heat will be transferred to the SMP layers, causing SMP to change its shape from the programmed configuration to the original configuration. As SMP the layers change their shape, the flexible CNP will deform with the SMP.

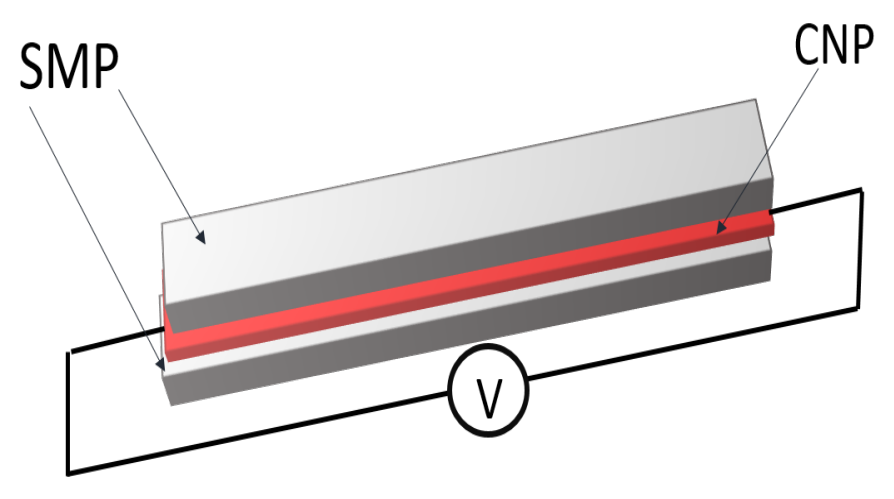

Figure 3.1: Concept model of CNP/SMP composite.

The shape recovery response of the composite under the application of a voltage difference requires a solution to a multiphysics problem involving electrical, thermal and structural behaviors. The design and optimization of deployable space structures employing SMP components can be attained by understanding the relations between the governing equations and material parameters.

In this section, the equations governing the shape recovery of the CNP/SMP composite are pre- 
sented. The behavior of the composite poses highly coupled partial differential equations. In this study, the approach is to investigate the governing equations in individual layers, while preserving the interaction properties between these layers.

\section{Electro-thermal Analysis}

Electro-thermal analysis is presented in this section in order to establish relations between electrical input and temperature field. Polymer is assumed to be insulative since the electrical conductivity of most polymers fall in range of $10^{-16}$ and $10^{-12} \mathrm{~S} / \mathrm{m}$ [67], compared to value of several hundred $\mathrm{S} / \mathrm{m}$ for CNP.

Considering the CNP layer, the energy balance is the governing equation. Under the application of voltage $\mathrm{V}(\mathrm{t})$, the CNP layer heats up and heat transfer occurs between the CNP layer and SMP layers. The energy balance for the CNP layer is given by Eq. (3.1),

$$
\dot{E}_{\mathrm{gen}}=\dot{Q}_{\mathrm{loss}}+\dot{E}_{\mathrm{stored}}
$$

where $\dot{E}_{\text {gen }}$ is the rate of energy dissipated in the CNP, $\dot{Q}_{\text {loss }}$ is the rate of heat transferred to SMP and surroundings, $\dot{E}_{\text {stored }}$ is the rate of energy stored in the CNP layer.

Assuming the heat generation to be uniform in the CNP layer, the heat generation rate is given by Joule's first law,

$$
\dot{E}_{\mathrm{gen}}=\frac{V^{2}}{R_{C N P}}=I^{2} R_{C N P}
$$

where I is the electrical current passing through the CNP layer and $R_{C N P}$ is the electrical resistance 
of the CNP layer, which can be stated as,

$$
R_{C N P}=\frac{L \vartheta}{A_{c}}
$$

where $\vartheta$ is the electrical resistivity of the CNP layer, L is the length of the CNP layer, $A_{c}$ is the cross sectional area which the current flows through. Now, the current density $\mathbf{J}$ is introduced as

$$
J=\frac{I}{A_{c}}
$$

Replacing the electrical current $I$ in Eq. (3.2) with the aid of Eq. (3.4) gives the heat generation rate in terms of the current density

$$
\dot{E}_{\text {gen }}=J^{2} \vartheta A_{c} L
$$

It is important to note that, generally, the temperature field in a material is dependent both on time and location, which adds complexity to the solution. The chosen approach to overcome this difficulty is the method of lumped mass heat transfer which assumes the temperature field to be uniform in CNP at any time. Thus, the temperature will be a function of time in the CNP layer. Consequently, the partial differential equation of heat transfer is reduced to ordinary differential equation. To be able to verify the lumped mass assumption, the thermal resistance at the CNP/SMP interface must be much higher than the thermal resistance inside the CNP layer.

Considering Figure 3.2, the ratio of thermal resistance at the interface and inside the CNP layer is defined as follows,

$$
\psi=\frac{R_{\mathrm{CNP}}^{T}}{R_{\text {int }}^{T}}
$$


where $R_{\mathrm{CNP}}^{T}$ represents the internal thermal resistance in the CNP layer, and $R^{T}$ int represents the interfacial thermal resistance. Interfacial thermal resistance is mainly explained by phonon scattering at the CNP-SMP interface [66]. If the value of $\psi$ is small, the lumped mass heat transfer solution is considered to be valid. This condition may be satisfied with high interfacial thermal resistance or low internal thermal resistance of CNP. Internal thermal resistance is inversely proportional to thermal conductivity. So, a thin CNP layer should be used and thermal conductivity of the CNP layer must be high. In that case, the temperature in the CNP layer can be asssumed uniform.

Heat loss term in Eq. (3.1) will generally contain heat loss due to conduction through the SMP boundaries, convective and radiative heat loss from the free boundaries. Neglecting the heat loss due to radiation, the rate of heat loss can be written as

$$
\dot{Q}_{\mathrm{loss}}=2 A_{\text {int }} \frac{T_{C N P}-T_{S M P, i}}{R_{\text {int }}^{T}}+A_{s, \operatorname{conv}} h\left(T_{C N P}-T_{\infty}\right),
$$

where $A_{\text {int }}$ is the contact area between SMP and CNP layers, $A_{s, c o n v}$ is the convective surface area of CNP, $h$ is the convection heat transfer coefficient, $T_{\infty}$ is the ambient temperature. $T_{C N P}$ is the temperature of CNP, and $T_{S M P, i}$ is the temperature of SMP layer at the interface. 


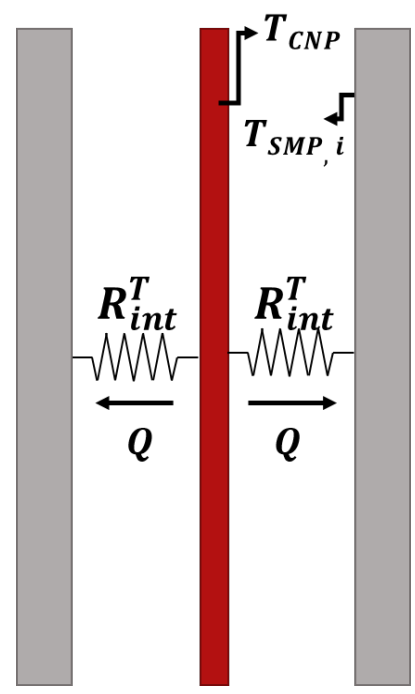

Figure 3.2: Schematics of heat transfer between layers.

Remaining fraction of dissipated heat will be stored in the CNP layer and causes the temperature of CNP to rise. Rate of energy stored in the CNP layer is written as

$$
\dot{E}_{\text {stored }}=\rho C_{p} A_{c} L \frac{d T}{d t}
$$

Substituting equations Eq. (3.2),Eq. (3.7) and Eq. (3.8) into Eq. (3.1), the governing differential equation can be obtained as

$$
J^{2} \vartheta A_{c} L=2 A_{\text {int }} \frac{T_{C N P}-T_{S M P, i}}{R_{\text {int }}^{T}}+A_{s, \text { conv }} h\left(T_{C N P}-T_{\infty}\right)+\rho C_{p} A_{c} L \frac{d T_{C N P}}{d t}
$$

As it can be seen from Eq. (3.7), the amount of transferred heat into SMP will depend on the interfacial thermal resistance between these two surfaces. In literature, interfacial thermal resis- 
tance between carbon nanotubes and polymer has been cited as a challenge [68] and attempts have been made by several research groups to account for this interfacial thermal resistance [69]. This thermal resistance is caused by phonon mismatch at the interface and depends on the amount of pressure between the layers. High thermal resistance between layers hinders the heat transfer into SMP, therefore affects the shape recovery behavior of the composite.

The purpose of analyzing the CNP layer is to model the amount of heat transferred into SMP. In order to do this, the temperature profile of the CNP needs to be obtained from Eq. (3.9). However, the solution also depends on the temperature of SMP at the interface. So, an iterative method is necessary to solve for the temperature of the CNP layer.

After solving for the temperature of the CNP layer, the heat transferred to SMP from the interface can be found from substituting $T_{C N P}$ into the first term of $\dot{Q}_{\text {loss }}$. Having determined the heat flux into SMP from the CNP layer solution, the temperature field in SMP needs to be solved. Temperature field solution in SMP is considered as Fourier conduction problem and stated by

$$
\alpha T_{\mathrm{xx}}=T_{\mathrm{t}},
$$

where $\alpha$ is the thermal diffusivity of SMP and T is the temperature field in SMP as a function of both time and location $(T(\chi, t)) . \alpha$ is given by the expression

$$
\alpha=\frac{k}{C_{p} \rho}
$$

where $k$ is the thermal conductivity of SMP, $C_{p}$ is the specific heat of SMP. The boundary conditions for this layer can be seen in Figure 3.3. 


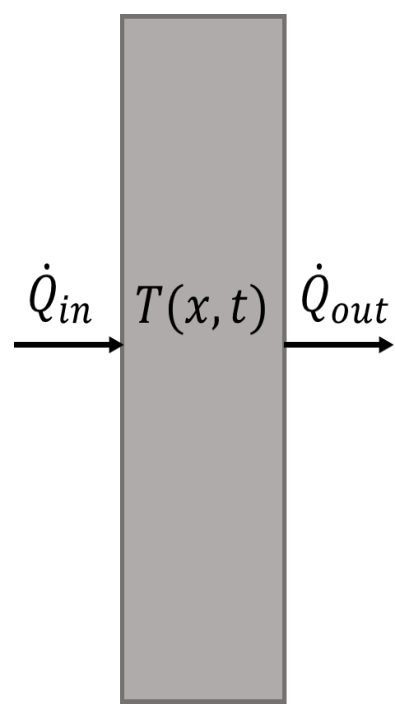

Figure 3.3: Heat transfer through shape memory polymer.

$\dot{Q}_{\text {in }}$ is the rate of heat transferred from CNP, and $\dot{Q}_{\text {out }}$ is the rate of heat transferred to the environment. Here, the boundary conditions are in derivative form, however, in general mixed boundary conditions can be present.

\section{Thermo-mechanical Analysis}

Shape memory polymers can undergo drastic shape changes under the application of stimuli such as light, heat and $\mathrm{pH}$. In this study, the investigated SMP is thermally activated. The means to apply heat is the CNP layer which is attached to SMP layers. The thermo-mechanical shape memory cycle consists of several distinct steps, namely programming, cooling, stowage and recovery. Programming step involves deformation of SMP at a temperature greater than activation temperature of SMP and holding at that deformation for a period of time. After programming, the next step is to cool SMP to a temperature below activation temperature while holding the deformation constant 
and then the deformation is released. Programmed and cooled SMPs can retain their shape during the stowage period and when external stimulus is applied, in this case it is heat due to Joule heating, they can recover their original configuration. A generic programming and activation of SMP can be seen in Figure 3.4.

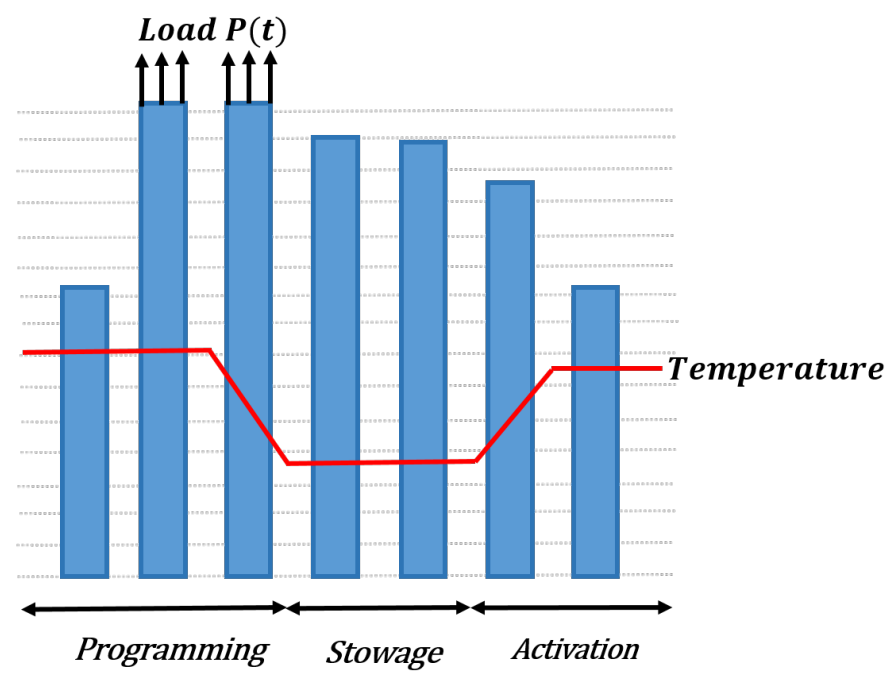

Figure 3.4: Programming and recovery of SMP.

Several constitutive models have been proposed in literature to capture the shape memory behavior of polymers but two approaches have been employed widely [26]. In the first model, the material consists of glassy and rubbery phases to capture shape memory behavior [70] while in the second modeling approach, viscoelastic properties of SMP have been used in conjunction with the time temperature superposition principle [36]. In this work, the latter modelling approach is used with Maxwell-Wiechert viscoelastic model. In this model, the material consists of different branches in parallel and in each branch, there is a spring and dashpot in series (Figure 3.5). 


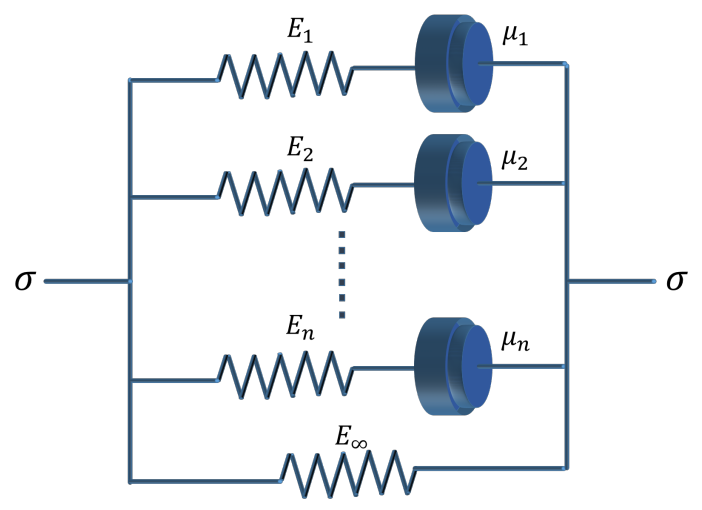

Figure 3.5: Generalized Maxwell model

To account for the long term response of material a free spring is added as another branch. Relaxation modulus of the material is represented as Prony series and given by

$$
E(t)=E_{\infty}+\sum_{i=1}^{n} E_{i} e^{-t / \tau_{i}}
$$

where $E_{i}$ are Prony series coefficients and $\tau_{i}(T)$ are relaxation times for each branch.

In linear viscoelasticity, stress in viscoelastic material is given by Boltzmann superposition integral

$$
\sigma(t)=\int_{0}^{t} E(t-\rho) \frac{d \varepsilon}{d \rho} d \rho .
$$

In the viscoelastic model for SMP, the viscosity of dashpots will decrease with increasing temperature, and material will show much lower resistance to deformation. When deformation is held at high temperature, the amount of force to maintain this deformation will decrease (i.e. stress relaxation). As shape memory polymer cools down, the viscosity of the dashpots will increase again 
and when the external load is removed, dashpots will show resistance to the springs internally. Therefore, the tendency of motion towards initial configuration will be obstructed.

To account for the effects of temperature, the time-temperature superposition principle (TTSP) is used. This principle employs a shift factor concept, which is the ratio of relaxation times at two different temperatures

$$
a_{T}=\frac{\tau_{i}(T)}{\tau_{i}\left(T_{0}\right)}
$$

For temperatures above the glass transition temperature, the shift factors are commonly determined from Williams-Landel-Ferry equation,

$$
\log \left(a_{T}\right)=\frac{-c_{1}\left(T-T_{0}\right)}{c_{2}+\left(T-T_{0}\right)}
$$

where $a_{T}$ is shift factor, $c_{1}$ and $c_{2}$ are material parameters, T is the temperature of SMP and $T_{0}$ is the reference temperature. For temperatures below the glass transition temperature, shift factors follow Arrhenius type equation

$$
\ln \left(a_{T}\right)=-\frac{E_{a}}{R}\left(\frac{1}{T}-\frac{1}{T_{0}}\right)
$$

where $E_{a}$ is the activation energy, $\mathrm{R}$ is the gas constant, $\mathrm{T}$ is the absolute temperature of SMP and $T_{0}$ is the absolute reference temperature.

In this work, the thermoviscoelastic model for small strains is used. The shape recovery formulation using spring dashpot elements have been adopted. The exact formulation of the shape recovery is a highly complex process. Qi et al. [36] included the derivation for shape recovery to prove their unified approach regarding programming and activation conditions using TTSP in 
their paper. That methodology is adopted and used as a basis for future discussions and the shape memory mechanism.

Considering the generalized Maxwell model for viscoelastic materials (Figure. (3.5)), for an individual branch, the stress is same for the spring and the dashpot elements for all times, i.e.

$$
\sigma_{\text {spring }}=\sigma_{\text {dashpot }}=E_{i} \varepsilon_{i}=\mu_{i} \frac{d\left(\varepsilon-\varepsilon_{i}\right)}{d t}
$$

where $\varepsilon_{i}$ is the strain carried by the spring element, $\varepsilon$ is the total strain also it is equal to strain carried by the free spring element. The difference between these two values will be carried by the dashpot element. For each branch, the ratio $\frac{\mu_{i}}{E_{i}}$ is defined as the relaxation time $\tau_{i}$ of that branch. From Eq. (3.18) and Eq. (3.14), the following relation can be written,

$$
\varepsilon_{i}=\left(\dot{\varepsilon}-\dot{\varepsilon}_{i}\right) \alpha(T) \tau_{i}
$$

The external stress carried by the generalized Maxwell element is given by,

$$
\sigma=E_{\infty} \varepsilon+\sum_{i=1}^{n} E_{i} \varepsilon i
$$

and the free recovery condition is stated as,

$$
\dot{\sigma}=E_{\infty} \dot{\varepsilon}+\sum_{i=1}^{n} E_{i} \dot{\varepsilon} i=0 .
$$


From Eq. (3.18) and Eq. (3.20), following equation can be written,

$$
E_{\infty} \dot{\varepsilon}_{i}+\frac{E_{\infty} \varepsilon_{i}}{\tau_{i}}+\sum_{i=1}^{n} E_{i} \dot{\varepsilon} i=0
$$

Expanding Eq. (3.21) for n non-equilibrium branches (shown for three non-equilibrium branches only) and rearranging them in matrix form,

$$
\begin{gathered}
E_{\infty} \dot{\varepsilon}_{1}+E_{1} \dot{\varepsilon}_{1}+E_{2} \dot{\varepsilon}_{2}+\ldots+\frac{E_{\infty} \varepsilon_{1}}{\alpha(T) \tau_{1}}=0, \\
E_{\infty} \dot{\varepsilon}_{2}+E_{1} \dot{\varepsilon}_{1}+E_{2} \dot{\varepsilon}_{2}+\ldots+\frac{E_{\infty} \varepsilon_{2}}{\alpha(T) \tau_{2}}=0, \\
E_{\infty} \dot{\varepsilon}_{3}+E_{1} \dot{\varepsilon_{1}}+E_{2} \dot{\varepsilon_{2}}+\ldots+\frac{E_{\infty} \varepsilon_{3}}{\alpha(T) \tau_{3}}=0, \\
{\left[\begin{array}{ccc}
E_{\infty}+E_{1} & E_{2} \\
E_{1} & E_{2}+E_{\infty} & E_{3} \\
E_{1} & E_{2} & E_{3}+E_{\infty}
\end{array}\right]\left[\begin{array}{l}
\dot{\varepsilon_{1}} \\
\dot{\varepsilon_{2}} \\
\dot{\varepsilon_{3}}
\end{array}\right]=\frac{-1}{\alpha(t)}\left[\begin{array}{lll}
\frac{1}{\tau_{1}} & 0 & 0 \\
0 & \frac{1}{\tau_{2}} & 0 \\
0 & 0 & \frac{1}{\tau_{3}}
\end{array}\right]\left[\begin{array}{l}
\varepsilon_{2} \\
\varepsilon_{3}
\end{array}\right] .}
\end{gathered}
$$

The problem is in the form of,

$$
[E]\{\dot{\varepsilon}\}=\frac{-1}{\alpha(t)}[\tau]\{\varepsilon\},
$$


and multiplying by the inverse of the modulus matrix, i.e. $[E]^{-1}$

$$
\{\varepsilon\}=\frac{-1}{\alpha(t)}[E]^{-1}[\tau]\{\varepsilon\}
$$

Eq. (3.27) can be normalized and transformed to decouple equations with transformed initial conditions for each branch. The final solution can be achieved as a function of the shift factors by transforming solution back to the time domain.

Considering the integral form, for viscoelastic materials, the governing relation between stress and strain is given by the Boltzmann integral,

$$
\varepsilon(t)=\int_{0}^{t} J(t-\rho) \frac{d \sigma}{d \rho} \mathrm{d} \rho
$$

Considering ideal stress relaxation conditions during the programming period of $t_{p}$ (i.e. deformation occurs at $\mathrm{t}=0$ instantly), for $0<\mathrm{t}<t_{p}$ stress is given by,

$$
\sigma(t)=E(t) \varepsilon_{0}
$$

When the external force is removed at $\mathrm{t}=t_{p}$, the stress change is modeled as a step function, i.e.

$$
\sigma=-\sigma_{0} H\left(t-t_{p}\right)
$$

This can be considered as applying a negative stress at the end of the programming cycle. The magnitude is the same as the stress level at the end of the programming cycle. Now, separating 
Eq. (3.28) into two parts,

$$
\varepsilon(t)=\int_{0}^{t_{p}} J(t-\rho) \frac{d \sigma}{d \rho} \mathrm{d} \rho+\int_{t p}^{t} J(t-\rho) \frac{d \sigma}{d \rho} \mathrm{d} \rho
$$

can be written. Noting that, first integral is representing the programming period, and Eq. (3.29) can be used to substitute $\sigma$, to yield

$$
\varepsilon(t)=\varepsilon_{0} \int_{0}^{t_{p}} J(t-\rho) \frac{d E(\rho)}{d \rho} \mathrm{d} \rho+\int_{t p}^{t} J(t-\rho) \frac{d \sigma}{d \rho} \mathrm{d} \rho .
$$

It can be seen that first integral involves the compliance and time derivative of the relaxation modulus in multiplication as integrand. Taking the Laplace transform of Eq. (3.28) and Eq. (3.13) yields,

$$
\begin{aligned}
& \bar{\varepsilon}(s)=s \bar{J}(s) \bar{\sigma}(s), \\
& \bar{\sigma}(s)=s \bar{E}(s) \bar{\varepsilon}(s) .
\end{aligned}
$$

Eq. (3.33) and Eq. (3.34) can be combined to provide the relation between the compliance and the relaxation modulus for viscoelastic materials in Laplace domain,

$$
\bar{E}(s) \bar{J}(s)=\frac{1}{s^{2}}
$$

Using the convolution theorem on Eq. (3.35), time domain relation between $\mathrm{E}$ and $\mathrm{J}$ can be found 
as [71],

$$
\int_{0}^{t} J(t-\rho) E(t) \mathrm{d} \rho=t .
$$

It can be seen that for viscoelastic materials, compliance and relaxation modulus are interrelated with convolution integral rather than reciprocality. Eq. (3.32) can be further simplified with the use of Eq. (3.36) and Eq. (3.30) to yield,

$$
\varepsilon(t)=\varepsilon_{0}-\sigma_{0} \int_{t p}^{t} J(t-\rho) \delta\left(t-t_{p}\right) \mathrm{d} \rho .
$$

where $\sigma_{0}$ is the stress level at the end of programming period. Finally Eq. (3.37) reduces to,

$$
\varepsilon(t)=\varepsilon_{0}-\sigma_{0} J\left(t-t_{p}\right) .
$$




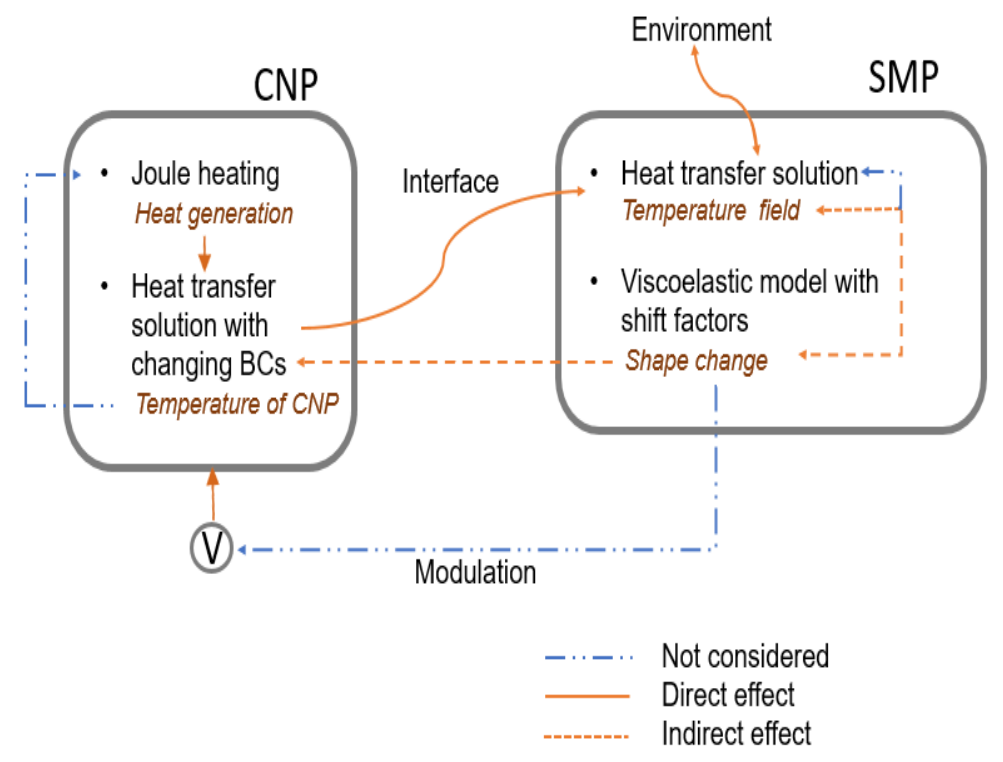

Figure 3.6: Solution approach and relations between governing equations.

Figure 3.6 visualizes the solution approach of this paper to solve this multiphysics problem. Formulations were made for Joule heating, heat transfer in the CNP and SMP. Heat transfer problem requires an iterative solution since the temperature field in SMP will also affect the heat transfer solution in the CNP. Temperature field in SMP is used with shift factor concept of TTSP to explain shape recovery behavior of SMP. The geometry change during recovery may alter the solution of the heat transfer. In general, material parameters such as density, thermal and electrical conductivities, specific heat are highly dependent on the temperature. Complex nature of boundary conditions results in nonhomogeneous PDEs and in most cases difficult to obtain an analytical solution. Therefore, a fully coupled electrical-thermal-structural FE model is developed and utilized to obtain the shape recovery behavior of CNP/SMP composite. 


\section{CHAPTER 4: MANUFACTURING}

For the viscoelastic characterization of SMP, specimens were manufacturing for testing. Dimensions of the test specimens were chosen to be $13 \mathrm{~mm}$ by $130 \mathrm{~mm}$ and a nominal thickness of 2.5 $\mathrm{mm}$. To create a mold for SMP, plexiglass of known dimensions (130 mm x $130 \mathrm{~mm}$ x $2.5 \mathrm{~mm}$ ) was cut and placed inside a horizontal mold. Then silicone from SmoothOn was poured into the pot and left for curing for 24 hours. The produced silicone mold was used for potting SMP. The larger dimension of the silicone mold allowed to manufacture multiple specimens in one manufacturing cycle. The silicone mold can be seen in Figure 4.1. Before using the silicone mold for SMP potting, silicone mold release spray was applied to the mold to increase the lifetime of the mold and to make the removal of SMP from the mold easier.

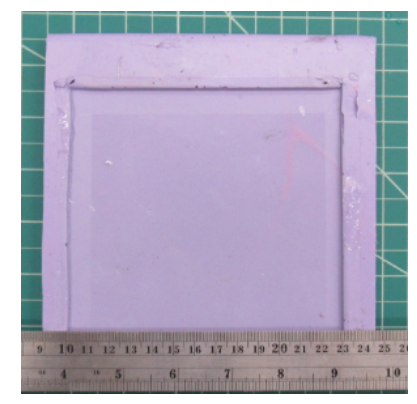

Figure 4.1: Mold for SMP potting

After obtaining the silicone mold, SMP components were weighted using an electronic scale with the mass ratio of 2:3 (16:24 grams) considering the volume of the mold. The components $\mathrm{A}$ and B of SMP were placed inside a degassing chamber (with Cps two stage vacuum pump) which was located inside an oven (Shel Lab SMO forced air oven) at $70^{\circ} \mathrm{C}$ (Figure 4.2). High temperature reduces the viscosity of the components, and makes degassing easier in vacuum environment. 
Materials were left for degassing for one hour.

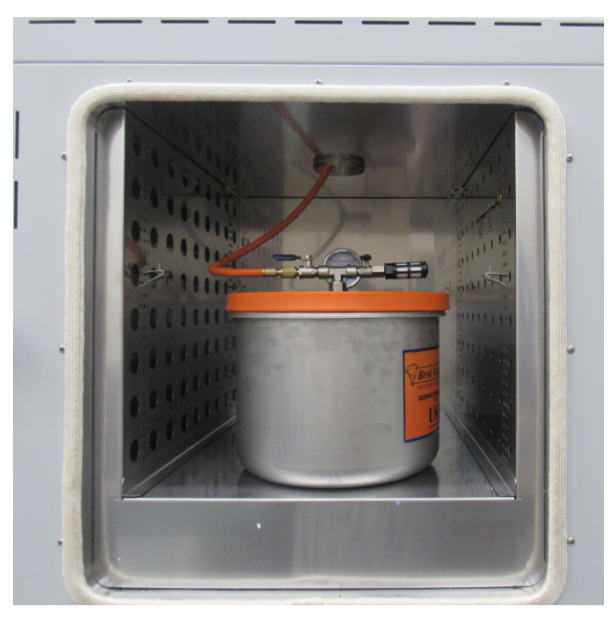

Figure 4.2: Degassing at $70^{\circ} \mathrm{C}$

Once degassed at $70^{\circ} \mathrm{C}$, the pressure inside was elevated to atmospheric pressure again and the materials were poured into same the container and mixed for 30 seconds at $60 \mathrm{rpm}$. After mixing the components, the SMP mixture was immediately poured into the silicone mold, to avoid gelation which was observed to occur within a minute. Polymerization reaction was considered to be completed 2 hours after onset of the gelation phenomenon.

The square block of SMP (130 mm x $130 \mathrm{~mm})$ was removed from the mold and cut into specimens using bandsaw. Specimen dimensions were measured for each specimen and unsatisfactory specimens (i.e. large variations in width and/or thickness) are removed from testing.

Manufacturing the composite posed a greater challenge since carbon nanopaper should be located inside the polymer, preferrably having two SMP layers of same thickness on each side. The previous silicone mold cannot be used in the manufacturing of the composite since it does not give control over the location of carbon nanopaper. One of the early concepts about the mold is pre- 
sented in Figure 4.3. Figure 4.4 shows gap in the mold for SMP to flow below the CNP.

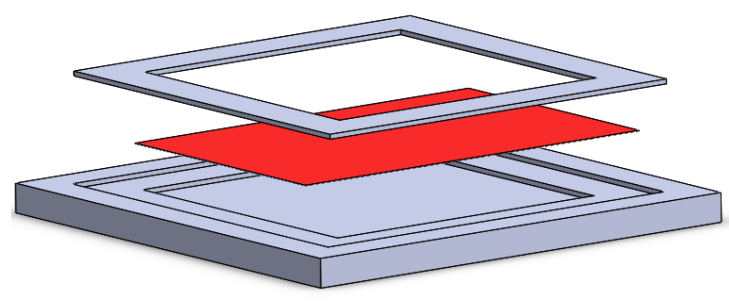

Figure 4.3: Horizontal mold exploded view

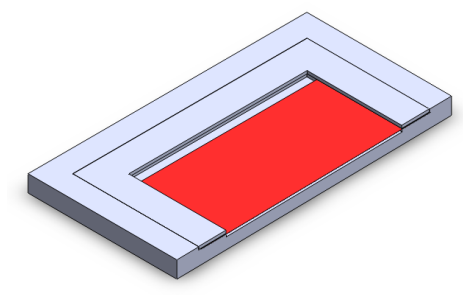

Figure 4.4: Horizontal mold cut view

The mold in Figure 4.3 requires the carbon nanopaper to be clamped between two parts of the mold such that it will be in tension to remove the waviness of the sheet. The carbon nanopaper does not cover the entire area of the mold, so two gaps on each side will allow polymer to flow on both sides of the carbon nanopaper. Although it was found promising, the specimens obtained using this mold were found to be unsatisfactory due to the deflection of carbon nanopaper layer during potting of the SMP. The CNP layer gets deflected once SMP fills the bottom layer, as a result, waviness and dry regions in the bottom of the CNP were observed.

Therefore, instead of using a horizontal mold, a vertical mold concept (where normal of the CNP 
layer is not collinear with gravity) is studied as illustrated in Figure 4.5. Two pieces of the silicone mold were produced, each having a step of one SMP layer $(2 \mathrm{~mm})$. This mold provides better control on the thickness of SMP layers. It is also possible to achieve non-uniform thickness between SMP layers as well as within one SMP layer (i.e. tapered, sinusoidal layers can be produced).

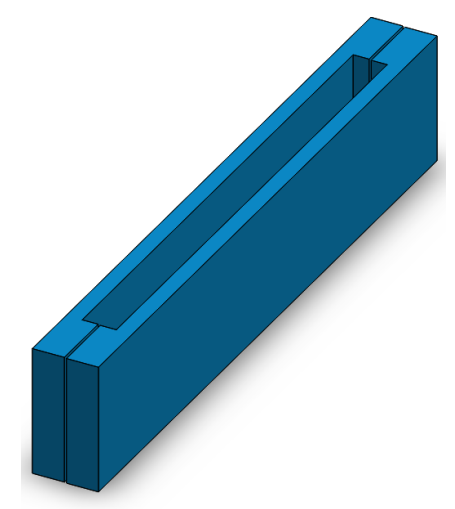

Figure 4.5: Vertical mold concept

Two pieces of the vertical mold were brought together and compressed using clamps, while the CNP layer is in between them in pre-tensioned condition. After mixing polymer components, the mixture was potted into the gap between silicone mold as illustrated in Figure 4.6. The result of this approach was found to be satisfactory and this mold was used to manufacture SMP/CNP composite. Highly uniform and controlled thickness was observed on each side with minimal waviness. Any excess polymer was removed from the sides and the final composite can be seen in Figure 4.7. 


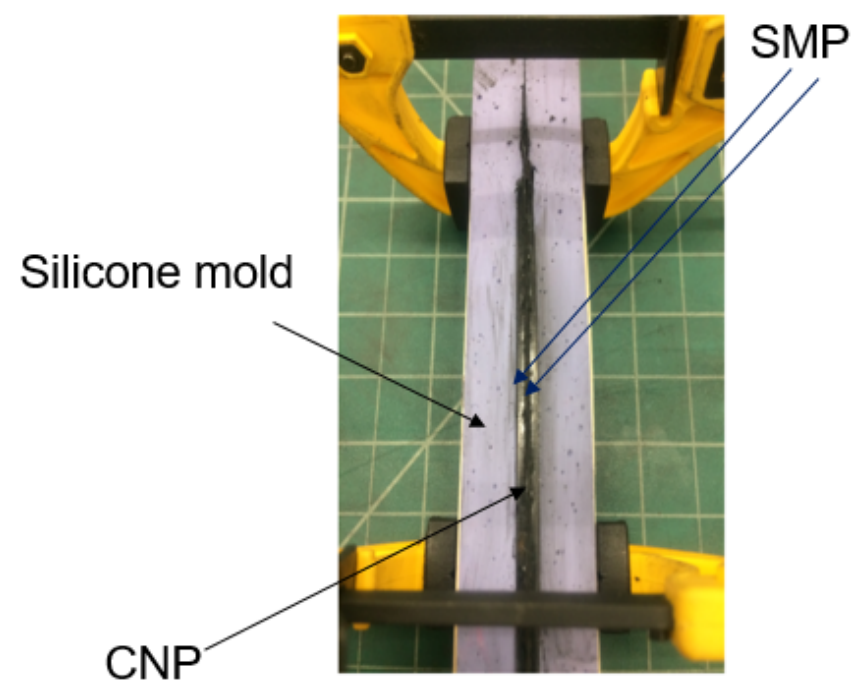

Figure 4.6: Final vertical mold concept

Figure 4.7: Front view of SMP/CNP composite

\section{Materials}

In order to study the validity of developed model and analyze the shape recovery behavior of the SMP/CNP composite, materials were purchased. For shape memory polymer, polyurethane based memory polymer MP-55 was purchased from SMP Technologies, Inc. MP-55 consists of resin and hardener, and available for potting.

For carbon nanopaper, Pyrograf III-HHT was purchased from Applied Sciences, Inc. PyrografIII is highly graphitic, and it consists of tubular carbon nanofibers (Figure 4.8 and Figure 4.9). 
HHT implies that it is high heat treated during the manufacturing process to remove iron catalysts efficiently, as well as to graphitize the fiber, resulting in highly graphitic carbon nanofiber and higher electrical conductivity [72]. The obtained carbon nanofibers have larger diameters (100 nm on average) than carbon nanotubes (1-10 nm).

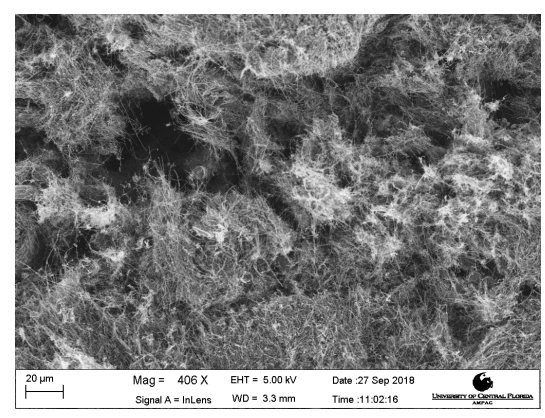

Figure 4.8: SEM image of carbon nanopaper, magnification 406X

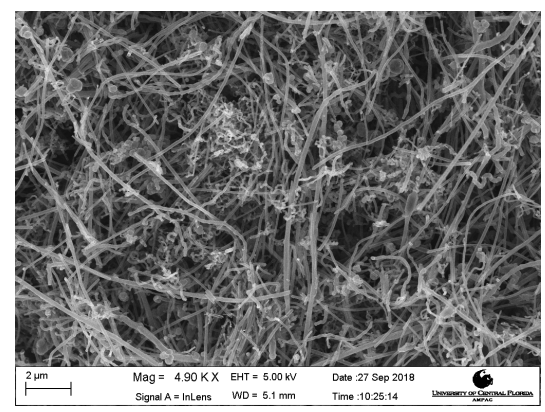

Figure 4.9: SEM image of carbon nanopaper, magnification 4900X 


\section{CHAPTER 5: CHARACTERIZATION OF CARBON NANOPAPER AND SHAPE MEMORY POLYMER}

The parameters and material properties that govern the shape recovery behavior of the SMP/CNP is presented in the Multiphysics Model section. In order to capture the shape recovery behavior accurately, the manufactured shape memory polymer has to be characterized. The multiphysics analysis has shown thermo-mechanical properties of SMP and electro-thermal properties of CNP plays an important role in the thermo-responsive behavior of the composite. In the next two sections, the characterization methodology of the shape memory polymer and carbon nanopaper is presented. In order to fully characterize material including chemical properties, Leng et al. [12] summarizes characterization tests, including Fourier transform infrared spectroscopy (FTIR) or Raman spectroscopy to identify chemical bonds present, scanning electron microscopy (SEM) to observe surface morphology.

Characterization of Shape Memory Polymer

In this study, as noted previously, the viscoelastic model approach is chosen to explain the shape recovery effect in conjuction with time-temperature superposition principle (TTSP). Therefore, tests for viscoelastic characterization of SMP are carried out.

In order to characterize the thermo-mechanical response of the polymers, two common approaches are widely used in the literature. The first approach is the dynamic mechanical analyzer (DMA) tests.

The second approach employs several stress relaxation tests conducted at different temperatures. For viscoelastic materials, the load to maintain constant deformation decreases over time. This 
phenomenon is called stress relaxation Figure 5.1. In that figure, ideal stands for deformation modeled as a step function and real stands for ramp loading.
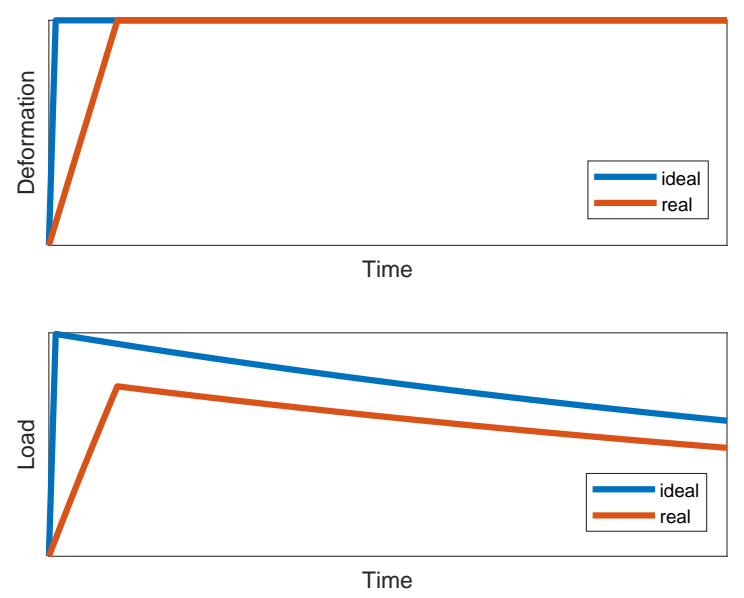

Figure 5.1: Stress relaxation

Stress relaxation tests setup can be seen in Figure 5.2. The test setup includes tensile test machine (MTS Criterion Model 43) with an environmental chamber (Thermcraft). Environmental chamber is used to test the specimen at higher temperatures. For strain measurement in the specimen, a digital image correlation system from Correlated Solutions is employed. 


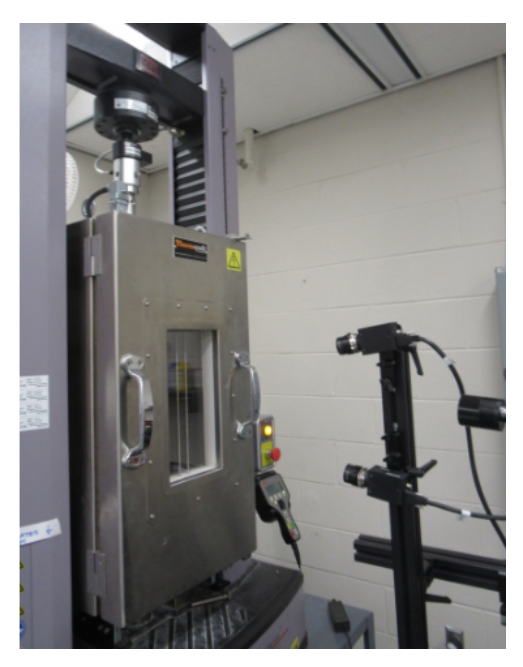

Figure 5.2: Stress relaxation test setup

First, the prepared specimens were attached to upper mechanical grips only and the temperature inside the chamber was elevated to desired test temperature (Figure 5.3). At this temperature, specimen was left for 20 minutes. This period helped to achieve uniform temperature field inside the chamber, and also allowed free thermal expansion of the extension rods and specimen. The effect of temperature drift of the load cell was minimized on the test data. After this period, the load reading was adjusted to zero and the chamber door was opened to attach the specimen to lower grips. Tightening of the grips caused the specimen to strecthn by a small amount, but with handset control the crosshead was adjusted so that the load reading remained zero. After attaching the specimen to the grips, gage length was measured before starting the test. The gage length was used to determine the crosshead displacement required in order to achieve desired strain. Another wait period of 15 minutes was given to reach steady state temperature field, and one final adjustment was made to the crosshead to ensure the specimen was load-free before the test. Using this procedure, thermal strain effects were nullified. 


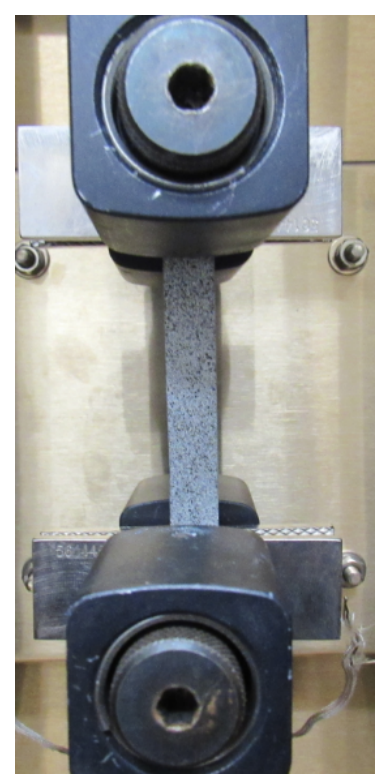

Figure 5.3: Test specimen attached to grips

Stress relaxation tests consist of two major stages: ramp loading until the desired deformation is reached and maintaining the desired deformation. The duration of the first period was approximately 10 seconds for each tests, and the stress relaxation behavior was observed for 30 minutes. Data acquisition frequency was set to $1 \mathrm{~Hz}$.

3D digital image correlation (DIC) was employed for all tests. DIC was used to correctly assess the strains in the material during testing. DIC gives information about strain field rather than a single value. For uniaxial test conditions, it is expected to see a uniform strain field on the sample.

After the tests, DIC pictures were analyzed via Vic-3D software to obtain the strain field in the specimen. The strain field was averaged over the area of interest and principal strains over time were recorded for all temperatures. Load and time data were taken from the MTS software. Combining with the constant strain value obtained from DIC, relaxation modulus (given by Eq. (5.1) ) 
can be calculated for each temperature.

$$
E(t)=\frac{\sigma(t)}{\varepsilon_{0}} .
$$

where $\varepsilon_{0}$ is the strain measured by DIC at the stress relaxation period, $\sigma(\mathrm{t})$ is the stress in the specimen as a function of time (obtained by dividing force reading with initial cross section area). It is important to note that, the initial period of data during constant strain period is omitted to limit the effect of the ramp loading on the data. The duration of omitted data is 10 times the duration of ramp loading period ( 100 seconds). Result of strain relaxation tests can be seen in Figure 5.4.

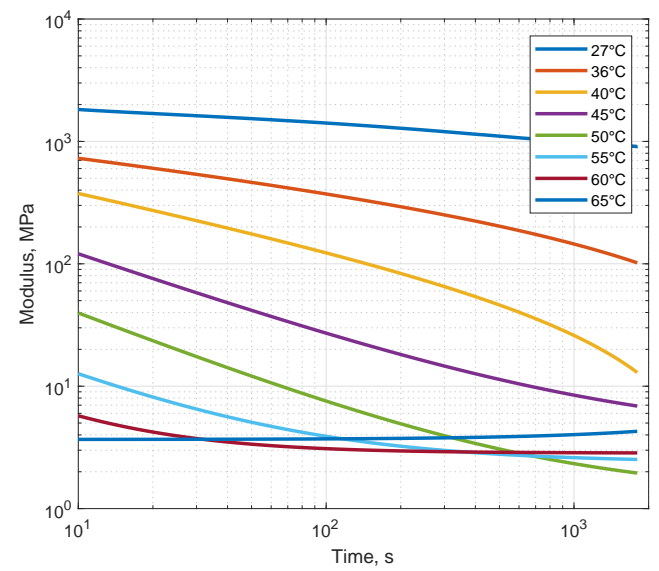

Figure 5.4: Stress relaxation test results

In order to achieve a master curve for the polymer, the time-temperature superposition principle is used. This principle suggests that modulus of the material can be equal at different time and temperature pairs, i.e.

$$
E(t, T)=E\left(t^{\prime}, T_{0}\right)
$$


where $T_{0}$ is the reference temperature, $\mathrm{t}^{\prime}$ is the reduced time. For example, the modulus value at $30^{\circ} \mathrm{C}$ after 10 minutes has the same value of the modulus at $45^{\circ}$ after 10 seconds.

The reference temperature for the masters curve was selected to be $27^{\circ} \mathrm{C}$. So, keeping the relaxation modulus at this temperature fixed, all other temperatures will be shifted horizontally in ascending temperature to form a continuous master curve. In order to achieve the horizontal shift, the results are first plotted in logartihmic scale, and different shift factors are specified and the shift factor that gives most overlap is accepted as shift factor. Experiments and shifting relaxation data is given in detail in Appendix D. It is important to note that, vertical shift which is given by Eq. (5.3) is neglected for this study,

$$
\frac{E(t, T)}{T}=\frac{E\left(t^{\prime}, T_{0}\right)}{T_{0}},
$$

where temperatures are in Kelvin.

In logarithmic scale, the shift factor has the effect of horizontal shift due to a change in time scale. If reference temperature $T_{0}$ is lower than shifted temperature, the shift factor will be less than unity, therefore in logaritmic scale, it will cause shifting to the right, which gives accelerated time response. If temperature is lower than $T_{0}$, shift factor is larger than unity, and when shifted, the curve will move left in logarithmic scale as given by Eq. (5.4)

$$
\begin{gathered}
t=\frac{t^{\prime}}{\alpha(T)}, \\
\log (t)=\log \left(t^{\prime}\right)-\log (\alpha(T)) .
\end{gathered}
$$


The resulting master curve at $27^{\circ} \mathrm{C}$ can be seen in Figure 5.5. A Prony series is fitted to the master curve using lsqcurvefit option in MATLAB which uses least squares regression scheme. Multiple Prony series coefficients can be obtained based on the chosen relaxation times, therefore, the relaxation times $\left(\tau_{i}\right)$ are fixed and curve fitting is done for corresponding Prony series coefficients. Relaxation times are chosen such that for every decade, there will be at least one relaxation time. Before fitting, the final value of master curve is assumed to be $E_{\infty}$ and subtracted from master curve to achieve better fit. The fitted master curve can be seen in Figure 5.5. Final relaxation times and corresponding relaxation moduli can be seen in Table 5.1.

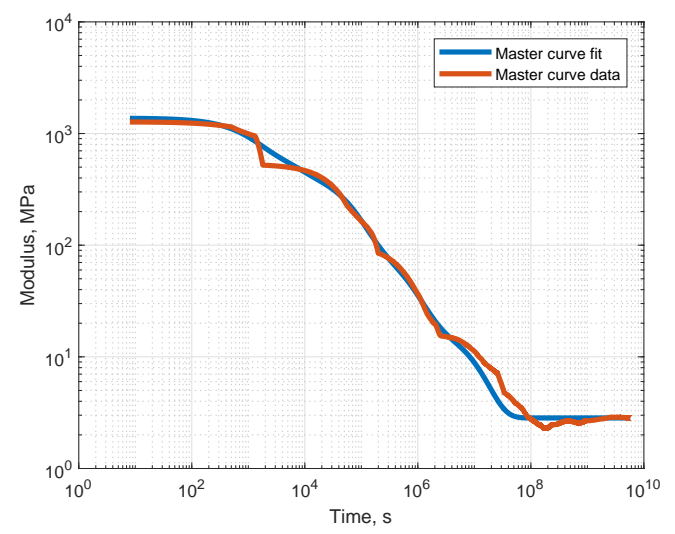

Figure 5.5: Master curve at $27^{\circ} \mathrm{C}$

The determined shift factors for temperatures are plotted in Figure 5.6. An analytical expression for shift factors is assumed in the form of the Williams-Landel-Ferry equation (Eq. (3.15)), and fitted to the shift factors data in order to determine coefficients $c_{1}$ and $c_{2}$ for the reference temperature of $T_{0}=27^{\circ} \mathrm{C}$. The fitting can be seen in Figure 5.6, and the fit results are presented in Table 5.2. 
Table 5.1: Prony series coefficients

\begin{tabular}{lr}
\hline$E_{i}, M P a$ & $\tau_{i}, s$ \\
\hline 604.11 & $1 \times 10^{3}$ \\
301.34 & $5 \times 10^{3}$ \\
252.94 & $5 \times 10^{4}$ \\
111.35 & $1 \times 10^{5}$ \\
56.87 & $5 \times 10^{5}$ \\
27.63 & $1 \times 10^{6}$ \\
16.41 & $1 \times 10^{7}$ \\
1.9 & $1 \times 10^{8}$ \\
2.84 & $E_{\infty}$ \\
\hline
\end{tabular}

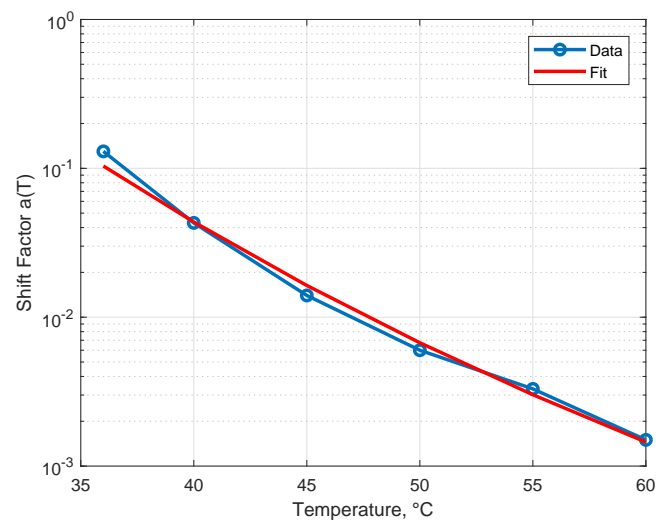

Figure 5.6: Shift factors 
Table 5.2: WLF Constants

\begin{tabular}{rr}
\hline$c_{1}$ & 9.66 \\
$c_{2}$ & 79.35 \\
$T_{0}$ & $27^{\circ} \mathrm{C}$ \\
\hline
\end{tabular}

Experimental challenges faced commonly include the strain measurement from DIC during the stress relaxation period. During stress relaxation period, the strain is fixed, however DIC results commonly display slowly changing strain during this period, especially at higher temperatures. This is mainly thought to be the effect of reflecting light on the cameras from the chamber, as well as heat waves in the chamber. It is reported that the heat waves can affect the performance of DIC for small strains. Also slippage occurs occasionally very fast during initial period of constant deformation period. By disregarding data spanning 10 times the ramping period, the effect of slippage is expected to be removed from the analyzed data.

Tests at lower temperatures (compared to $T_{g}$ ) has shown smaller strains compared to higher temperatures tests although the crosshead displacements were adjusted so that the resulting strains would be the same. The reason for this error can be explained via frictional forces between grips and specimen. For temperatures lower than $T_{g}$, specimens are glassy, making it harder to grip and deform with the crosshead. At higher temperatures, specimens are in rubbery state and it is easier to grip them and they can deform, specimen goes to the same deformation level.

The glass transition temperature $T_{g}$ is one of the most important parameters for polymer materials. One way to estimate the glass transition temperature is to check the relaxation modulus at a specified time for all temperatures. This modulus value is usually taken at 10 seconds (ten seconds modulus, $E_{10}$ ) or after 1 minute (one minute modulus, $E_{1}$ ) [71]. When $E_{1}$ is plotted against 
temperature, glass transition temperature can be constrained to the region where there is a drop in $E_{1}$. One minute modulus is plotted in Figure 5.7. A sharp drop in modulus values could not be observed, however it can be argued that $T_{g}$ lies within $40^{\circ} \mathrm{C}$ and $50^{\circ} \mathrm{C}$.

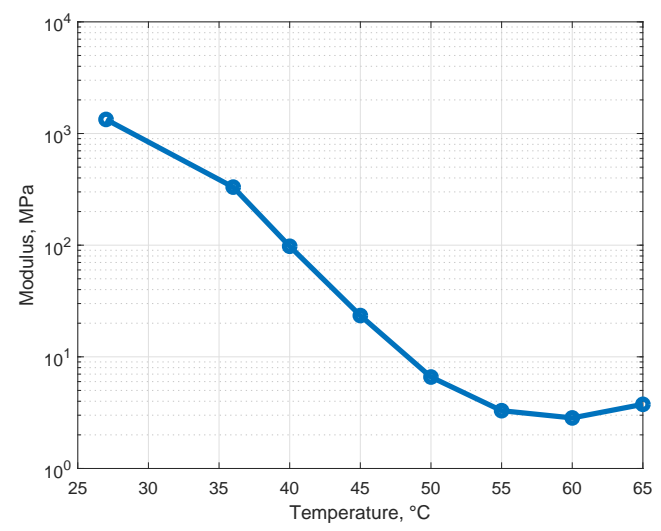

Figure 5.7: One minute modulus, $E_{1}$ at different temperatures

\section{Carbon Nanopaper Characterization}

For carbon nanopaper characterization, uniaxial tests are carried out to measure the elastic modulus of the material. Although the elastic modulus is an important mechanical property to consider, determining the failure strain of the carbon nanopaper is also needed to ensure the integrity of the carbon nanopaper during programming SMP/CNP composite.

Carbon nanopaper sample specimens with dimensions of $10 \mathrm{~mm}$ x $100 \mathrm{~mm}$ were cut from PyrografIII. Specimens and test setup can be seen in Figure 5.8 and Figure 5.9. Fracture patterns of the carbon nanopaper can be seen in Figure 5.10. 


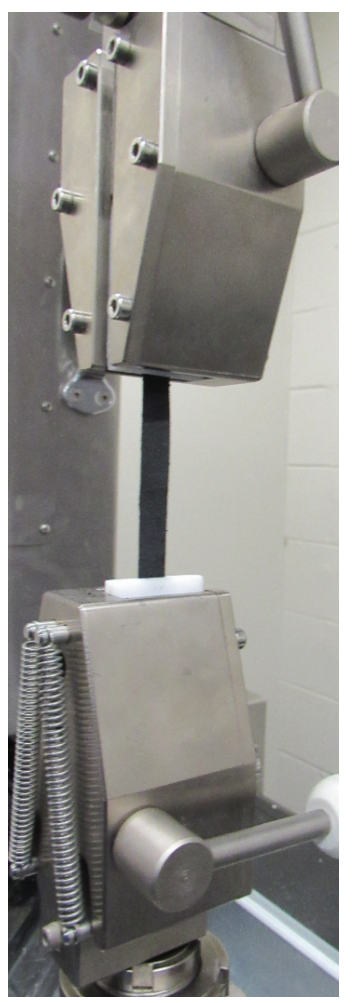

Figure 5.8: Test setup for uniaxial tests of Carbon Nanopaper

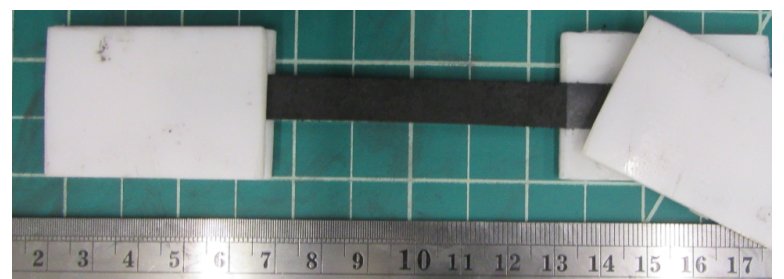

Figure 5.9: Carbon Nanopaper specimen preparation 


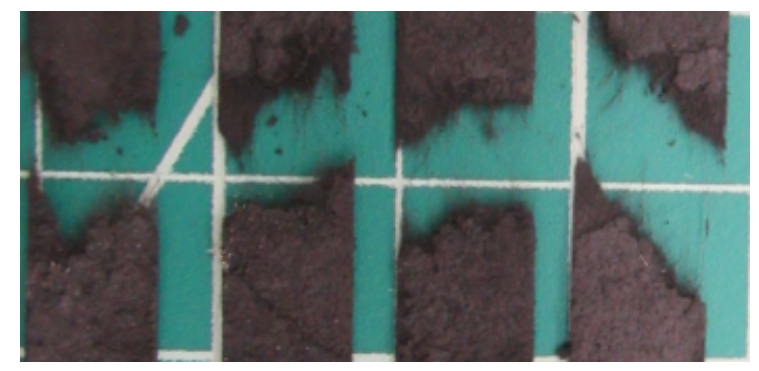

Figure 5.10: Fracture patterns of Carbon Nanopaper

In fracture tests, information about failure strain and elastic modulus can be extracted. For CNP, $1 \%$ strain can be accepted as the failure strain (Figure 5.11). Early failure of the third specimen can be attributed to previously existing damages due to handling. In Figure 5.12, the hysteresis behavior can be observed in cyclic deformation. From Figure 5.12, it is clear that first loading path gives the highest stress and following cycles are highly repeatable without a noticable loss. This result can be interpreted as preconditioning of the CNP. The first deformation path changes the configuration of fiber network, and it is much less pronounced in following deformation cycles.

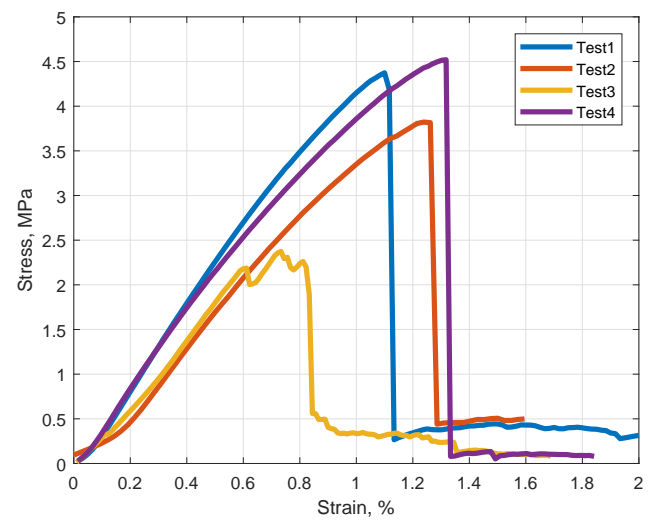

Figure 5.11: Carbon nanopaper tension tests 

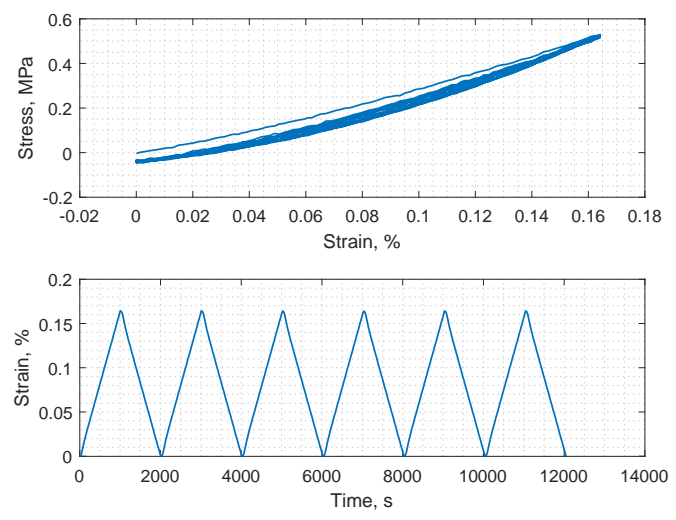

Figure 5.12: Hysteresis in cylic deformation

For complete characterization, additional tests can be run on SMP such as differential scanning calorimetry (DSC) to obtain glass transition temperature and melting temperature of crystalline regions (if present). DSC setup can apply constant and equal temperature ramp to polymer and reference material and monitor the heat needed to achieve that temperature ramp (power-compensation DSC). At $T_{g}$, second order transition occurs (i.e. change in heat capacity). Heat capacity of polymer above $T_{g}$ is higher and transition of specific heat over temperature gives information about $T_{g}$

For SMP, constant specific heat capacity is assumed, but it is known that specific heat capacity for all polymeric materials is dependent on temperature [73] and heat capacity can be approximated as two linear functions of temperature with discontinuity at $T_{g}$ [74]. Discussion of density and other thermal properties such as thermal conductivity of polymer as a function of temperature can be found in literature [75] as well as their nanocomposites [68]. Generally, polymers display an increase in thermal conductivity at temperatures up to $T_{g}$, and decrease with increasing temperature above $T_{g}[76]$. 


\section{CHAPTER 6: FINITE ELEMENT MODEL}

As discussed in previous sections, the shape recovery behavior of composite poses highly coupled PDEs. To be able to solve this multiphysics problem, a nonlinear finite element model is developed and analyzed using ABAQUS software. Fully coupled electrical-thermal-structural analysis has been employed to obtain solutions for time dependent temperature, stress and strain fields simultaneously.

Dimensions of the composite model are $100 \mathrm{~mm} \times 10 \mathrm{~mm}$ x $2 \mathrm{~mm}$ for SMP layers and $100 \mathrm{~mm}$ x $10 \mathrm{~mm} \times 0.38 \mathrm{~mm}$ for the CNP layer. Layers are created as separate parts and then assembled together. The reason for creating separate parts is to be able to define interaction properties later. All parts are modeled as 3D and deformable. Also a reference point is created.

The meshed finite element geometry is given in Figure 6.1. 8-node trilinear displacement, electric potential and temperature elements (Q3D8) have been used in the model with approximate global size of $1 \mathrm{~mm}$, yielding 1000 elements in the CNP layer and 4000 elements in the SMP layers.

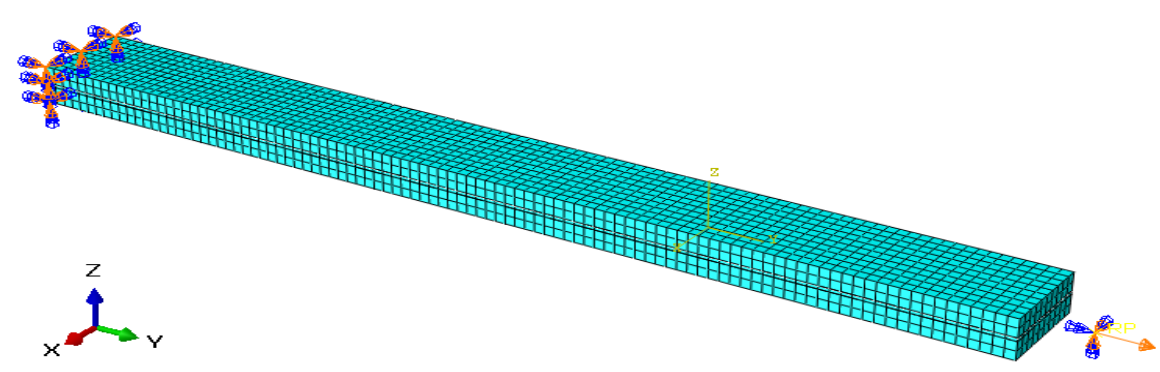

Figure 6.1: Meshed geometry of composite.

The material properties required in order to carry out this FEA have been determined from the the- 
oretical discussion of this multiphysics problem. Material properties of CNP and SMP have been assigned. Parameters used for each material are tabulated in Table 6.1 and assumed parameters are marked. The density of CNP was calculated from the areal weight value given by the manufacturer. The CNP layer was modeled as solid, homogeneous and elastic, while SMP was modeled as viscoelastic with Williams-Landel-Ferry material constants to capture the temperature dependent behavior of SMP.

Table 6.1: Material parameters for FE model

\begin{tabular}{cccc}
\hline Parameters & SMP & CNP & Unit \\
\hline Thermal Conductivity & 0.15 & 800 & $\frac{\mathrm{W}}{\mathrm{mK}}$ \\
Electrical Conductivity & $0.001^{*}$ & 666 & $\mathrm{~S} \cdot \mathrm{m}$ \\
Coefficient of Thermal Expansion * & $5 \mathrm{E}-5$ & - & $1 / \mathrm{K}$ \\
Joule Heat Fraction & 1 & 1 & - \\
Specific Heat & $2000^{*}$ & 710 & $\frac{\mathrm{J}}{\mathrm{kgK}}$ \\
Density & $860 *$ & 105.263 & $\frac{\mathrm{kg}}{\mathrm{m}^{3}}$ \\
Poisson's ratio & 0.45 & $0.33 *$ & - \\
$E_{\infty}$ & 2.84 & 300 & $\mathrm{MPa}$ \\
\hline
\end{tabular}

This FEA consists of 5 steps, in the order of, the prescribed deformation at high temperature, maintaining deformation at high temperature, cooling down to a temperature below activation temperature, removing the load and stow, and applying voltage difference to CNP layer.Durations of these steps are 1, 100, 3600, 1000 and 1000 seconds, respectively. These steps fully represent a typical thermo-mechanical cycle for SMPs.

The nonlinear geometry option was activated for all these steps. For the initial deformation step, 
viscoelastic procedure was chosen. For the remaining of the steps, coupled thermal-electricalstructural analysis was carried out. For all steps creep/ swelling/ viscoelastic behavior wass included and CETOL was set to 0.01 .

Contact interactions between surfaces were defined in order to capture the effect of non-material parameters. CNP surfaces were assigned as master surfaces and corresponding SMP surfaces were assigned as slave surfaces with no adjustment. As contact properties, tangential behavior, normal behavior, thermal conductance and electrical conductance were chosen. Tangential behavior of contact was formulated as rough which makes sure slip will not occur at the interface, therefore securing perfect mechanical bond. Normal behavior was specified as hard contact and separation after contact was not allowed. These contact properties made sure CNP and SMP layer deform together at all times.

Thermal conductance and electrical conductance properties at the interface were specified with respect to clearance only. Electrical conductance was assumed zero at the interface irrespective of clearance since electrical conductivity of SMP is negligible compared to CNP. So there will be no current passing through SMP layers. For thermal conductance, $1 \times 10^{5}$ is assumed at zero clearance. The actual value of interfacial thermal resistance/conductance is difficult to obtain. Han et al[68] gives an average value of $1 \times 10^{8} \mathrm{Wm}^{-1} \mathrm{~K}^{-1}$ for carbon nanotube polymer interaction.

In simulations, starting from the cooling down step, a surface film condition was created. A surface film condition coefficient was assumed on the same order of magnitude with natural air convection coefficient. A uniform sink temperature was chosen as $30^{\circ} \mathrm{C}$.

Finally, a kinematic coupling constraint was used to couple the reference point and top surface of the composite (Figure 6.1). Only longitudinal displacement and rotational degrees of freedom were constrained. 
In this $\mathrm{FE}$ model, the composite was deformed $1 \mathrm{~mm}$ uniaxially at $60^{\circ} \mathrm{C}$, which corresponds to $1 \%$ strain. Displacement boundary condition was specified at the reference point. At the other end of the composite, all degrees of freedom were restricted. The temperature of the composite will remain constant during the hold period. With the start of the cooling step, the temperature field was calculated for every increment. After the convective cooling of the composite to $30^{\circ} \mathrm{C}$, the displacement boundary condition specified at the reference point was deactivated (at the beginning of the stowage step). After this point, there was no external mechanical load applied to the FE model. At the beginning of activation, an electrical potential boundary condition was specified at the ends of the CNP layer. 


\section{CHAPTER 7: SHAPE RECOVERY EXPERIMENTS OF CNP/SMP COMPOSITE}

Experiments are carried out on CNP/SMP composite to evaluate the performance of the proposed FEA model. The MTS Criterion Model 43 EM Tensile Test machine is used to create a test template to fully observe the thermo-mechanical cycle of the composite. The experimental setup is shown in Figure 7.1. Two K-type thermocouples have been used, one was taped to the middle of the composite, the other one was taped to the lower grip close to the specimen. For data acquisition from thermocouples, NI cDAQ-9185 is used with NI 9219 module and LabView2018.

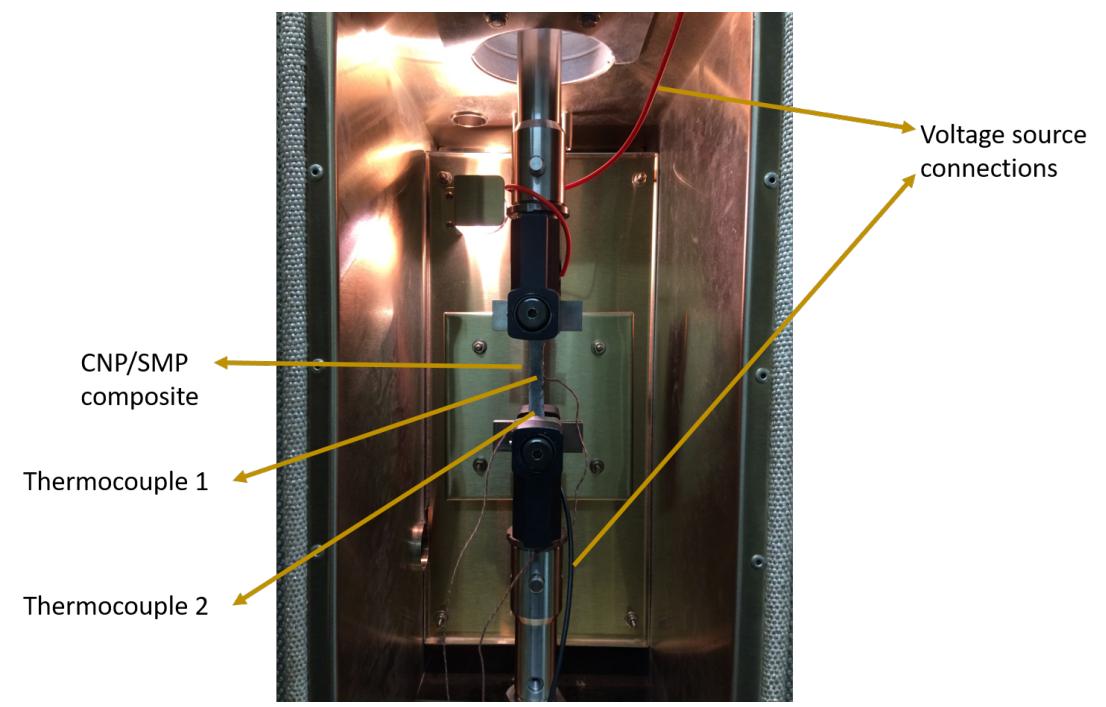

Figure 7.1: Shape recovery experiment setup.

Initially, the composite was attached to the upper grip only and environmental chamber temperature was increased to $60^{\circ} \mathrm{C}$. This allowed the composite to expand freely. After that, the composite was attached to the lower grips. Wires of the voltage source were connected to CNP extensions of the composite (red and black cables, for upper and lower ends, respectively). To ensure there 
was minimal load initially, the crosshead was allowed to adjust itself to maintain zero load. After thermal equilibrium was reached, the composite was deformed $1 \mathrm{~mm}$ uniaxially in 10 seconds. Deformation was maintained at $60^{\circ} \mathrm{C}$ for 100 seconds and then environmental chamber door was opened to cool the composite via natural convective cooling at deformed configuration. The duration of the cooling step was one hour. After one hour of natural cooling, load was reduced to zero in approximately 3 seconds. The crosshead was allowed to move to maintain zero load afterwards. Stowage characteristics were recorded for 1000 seconds. In the free recovery step, a voltage controlled DC power source was turned on at $20 \mathrm{~V}$ to observe free shape recovery response of the composite for 500 seconds. The crosshead displacement value was taken from MTS for the duration of the experiment. The strain evaluation was based on crosshead displacement and gage length. Due to complexity of test setup, DIC was not used for these experiments. During shape recovery tests, data acquisition rate was set to $1 \mathrm{~Hz}$.

Shape recovery experiments have several steps in order to fully encompass thermo-mechanical cycle for SMP composite (ie. programming, stowage, activation). Designing this experiment allowed to measure forces and deformations over time quantitatively. The main challenge for these experiments were due to high number of the steps involved, some of them requiring user intervention (e.g. opening chamber door for natural cooling, turning on DC power source for activation). While creating the test template, switching from crosshead control to load control test introduced some problems as well. Load control (i.e. fixing the load while crosshead is to move accordingly) was achieved by assuming PID parameters of soft material and by allowing a load decrease rate of $5 \mathrm{~N} / \mathrm{s}$. 


\section{CHAPTER 8: RESULTS AND DISCUSSION}

It is essential to compare experimental results with finite element analysis results to see the performance of the model in capturing the composite behavior. For that purpose, in FE model, boundary conditions are modified such that, they will represent actual programming conditions as close as possible. For this purpose, convective heat transfer coefficient is set to $1 \mathrm{Wm}^{-2} \mathrm{~K}^{-1}$ for the duration of cooling and stowage, and modified to $30 \mathrm{Wm}^{-2} \mathrm{~K}^{-1}$ in activation step to match the thermocouple readings in experiment. Comparisons of the FE model with experimental results can be seen in Figure 8.1 and Figure 8.2.

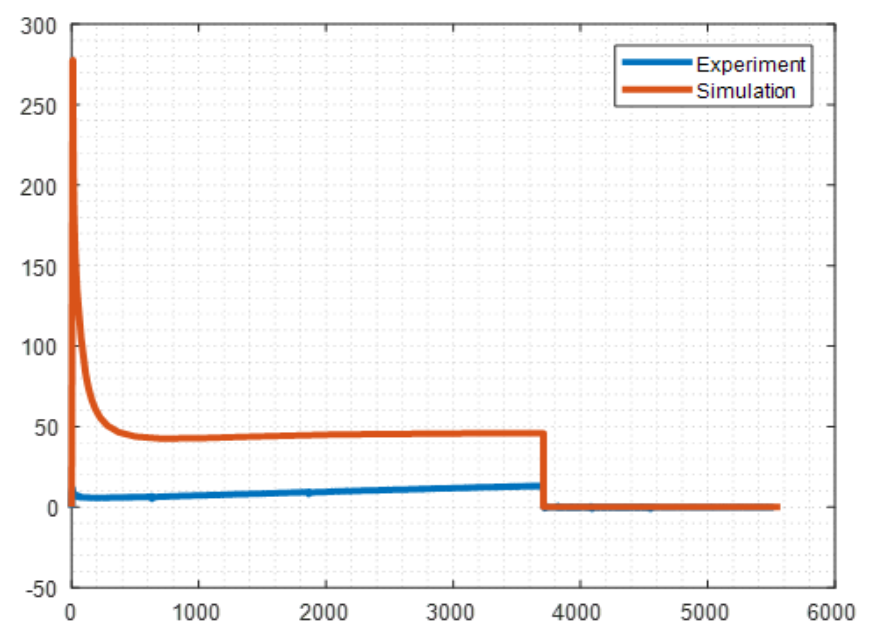

Figure 8.1: Comparison of external force of FE model and experiment.

The finite element model highly overpredicts the force during constant deformation and cooling steps. Although the force drastically reduces within a very short timeframe with the start of the constant deformation step, overprediction remains. Experimental results show negligible stress relaxation at $60^{\circ} \mathrm{C}$. During cooling, forces in both experimental and $\mathrm{FE}$ results display increasing 
trends. This increase in force can be explained as an effect of the coefficient of thermal expansion. The compsosite specimen tries to contract as it cools down but, is hindered by fixed crosshead displacement. The forces are reduced to zero by the end of the cooling step, and remained zero for the rest of the test.

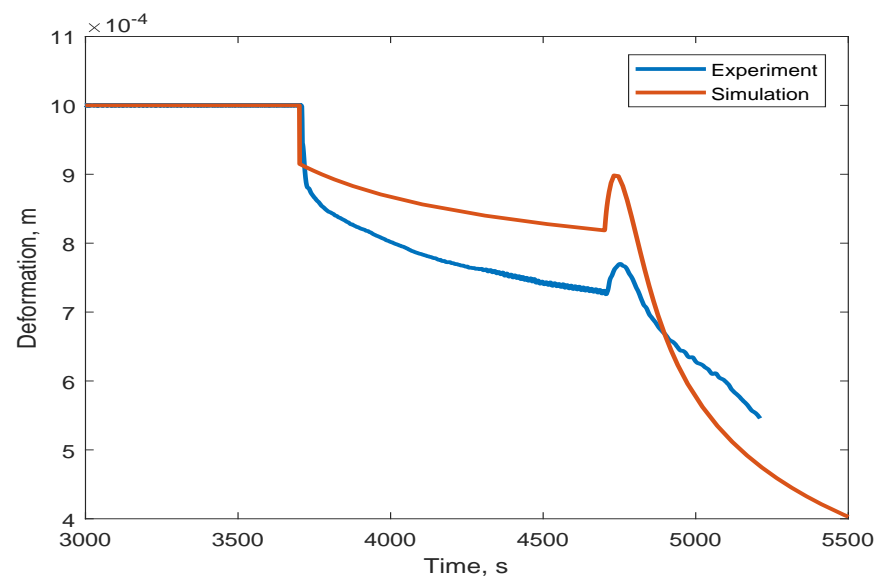

Figure 8.2: Comparison of shape recovery of FE model and experiment.

Considering the deformation of the composite in Figure 8.2, there is a sudden shape recovery when the external force is removed. The magnitude of the springback effect is closely related to effective modulus of the composite at that instant and the amount of force. The finite element model predicts $91 \%$ of the initial deformation will be preserved when the external load is removed. In experiment, the value is about $87 \%$. Ideally, during the stowage period there should be no shape recovery. However, the specimen has shown a considerable shape recovery during stowage, the deformation is reduced to $73 \%$ of the programmed deformation after 1000 seconds. The finite element model also shows a shape recovery during stowage around $30^{\circ} \mathrm{C}$. The discrepancy between these results can be explained with inadequately modeled experimental conditions.

In the experiment, the grips are always in contact with the specimen for the duration of the test. Fig- 
ure 8.3 shows thermocouple readings with simulation result for the temperature value at midpoint node located on the surface of the model. Thermocouple readings show large variation during the cooling step. The first thermocouple which was located in the middle of composite, cooled down much faster than the thermocouple located on the grip. The reason is that thermal capacitance of the extension rods and grips are much higher than thermal capacitance of the composite, therefore, they are harder to cool. Referring to Figure 8.3, this also means that as the composite cools down, there will considerable heat transfer from grips to composite, which will affect the shape fixity of the composite.

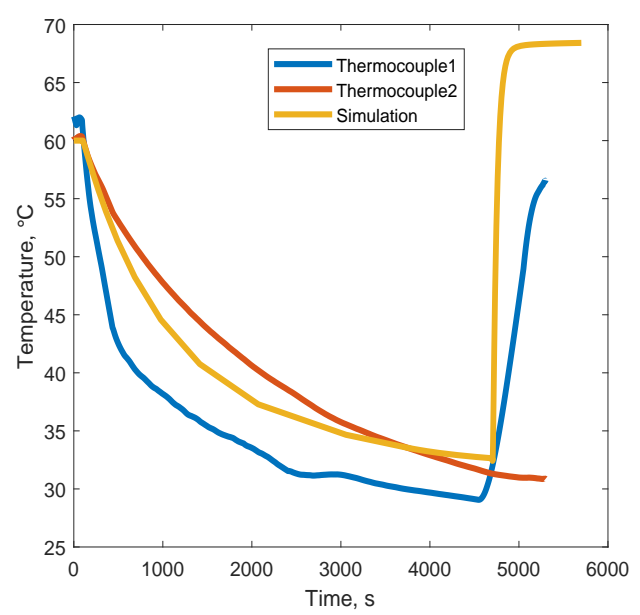

Figure 8.3: Temperature data from thermocouple readings and simulation.

In Figure 8.3, when the DC power source is turned on the end of the experiment, surface temperature of the composite increases drastically as expected and converges to a constant value. The same temperature profile is captured in the finite element simulation. However, the finite element simulation predicts a faster temperature rise and higher converged temperature than the experiment. This deviation can be used to explain the difference in the shape recovery step.

During the activation step, experimental results and the finite element model exhibit agrees qual- 
itatively. When the voltage source is activated, the composite starts to heat up. At first, instead of going back to original configuration directly, the composite expands solely due to thermal expansion. While this expansion takes place, also shape recovery starts. The deformation profile observed is the combined result of these two effects. Although temperature keeps rising, the expansion of composite first slows down since shape recovery effect starts to become more dominant. There is a point of unstable equilibrium where expansion of the composite reaches a maximum. At this point, the effect of the coefficient of thermal expansion is nullified by shape recovery. After this point, shape recovery takes over and the composite starts to return to its original shape. In the finite element model, different voltage values are used in activation step, and shape recovery behavior in each case is plotted in Figure 8.4 during activation. As it can be seen from Figure 8.4, the magnitude of expansion depends on the heating rate during activation. Higher actuation voltages result in faster heating and shape recovery but also higher expansion peaks.

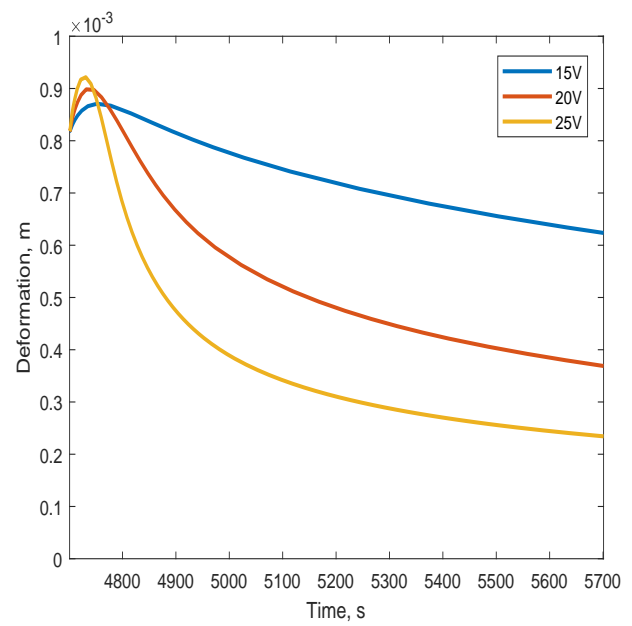

Figure 8.4: Shape recovery with different actuation voltages.

Figure 8.5 shows temperature field in a cut view of the composite during heating. A non-uniform 
temperature field in the composite is present. The highest temperature is at the CNP layer and the corners of the specimen have the lowest temperature due to larger convection surface. Figure 8.6 displays the stress field in the composite during stowage. Although there are no external loads present at this step, the composite is internally stressed. The CNP layer is under tensile stresses and SMP layers exhibit compressive stresses.

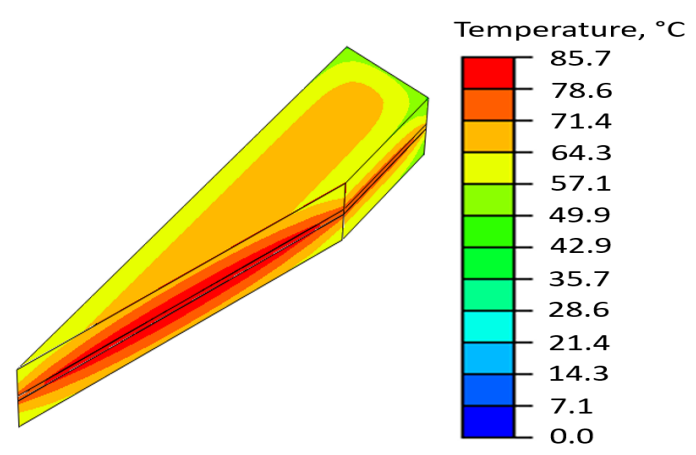

Figure 8.5: Temperature field during heating $(\mathrm{t}=240 \mathrm{~s})$.

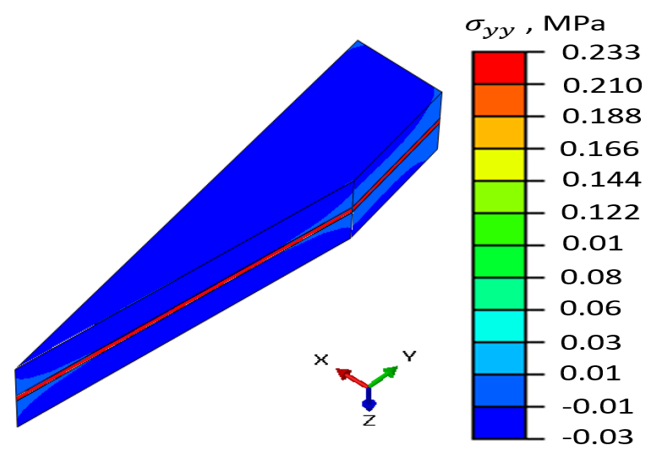

Figure 8.6: Stress field at stowage $(\mathrm{t}=403 \mathrm{~s})$. 
In Figure 8.7 and Figure 8.8, results of the finite element simulation are plotted. Figure 8.7 shows load and deformation history of the composite. In Figure 8.8, the thermo-mechical cycle is presented for the same simulation. In these figures, full recovery line marks the line of zero force and zero deformation (original shape with no external loads) in 3D.

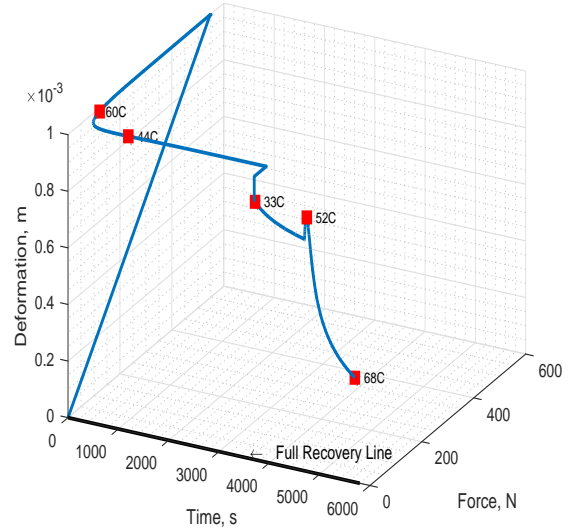

Figure 8.7: Load and deformation history.

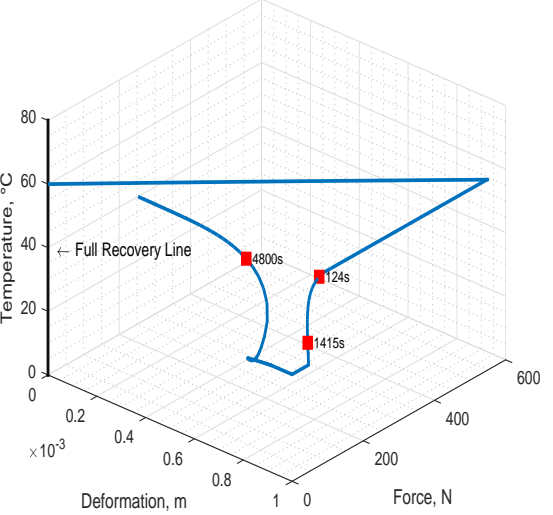

Figure 8.8: Thermo-mechanical cycle of composite. 


\section{CHAPTER 9: CONCLUSIONS}

In this work, the shape recovery behavior of uniaxially programmed, electrically activated CNP/SMP composite is studied. A solution approach regarding the multiphysics of the problem is presented. Following this approach, governing equations and their relations are derived and important parameters are noted. A fully coupled electrical-thermal-structural finite element model is developed. The parameters defining shape recovery response of the composite are not limited to material properties, they also include the parameters coming from the interface of the layers. Therefore, contact properties between CNP and SMP are preserved in the deveoped finite element model.

In order the validate the developed finite element model, a composite consisting of a CNP layer sandwiched between two SMP layers was fabricated. The composite was tested to obtain programming and shape recovery behavior of CNP/SMP composite under uniaxially programmed conditions. It is found that, the developed FE model shows promising results and can capture the characteristics of strain, stress and temperature fields in the composite during programming, stowage and activation periods.

The shape recovery behavior of the CNP/SMP composite is observed for different actuation voltages. From design perspective, the proposed model can be used to study effects of material properties, as well as different composite configurations such as unequal SMP layer dimensions, CNP/SMP contact area, the locations of the CNP layers in composite, etc.

The main contributions of this study are the mathematical formulation of the underlying mechanism of electrically actuated shape memory composite, the fully coupled electrical-thermal-structural finite element model which can capture general characteristics of shape memory cycle of the composite. In the future, material parameters used in finite element simulations will be refined with thorough characterization of individual materials. 


\section{CHAPTER 10: FUTURE WORK}

The work presented in this thesis will be used as the framework to determine the shape recovery of SMP/CNP composites. To be able to use the developed model to actuate the composite as desired, sources of discrepancy reported in previous section between experiments and model need to be

eliminated. In the near future, rigorous shape recovery experiments need to be carried out together with the viscoelastic characterization of the composite, rather than characterization of the SMP.

After achieving good agreement quantitatively of the shape recovery experiments with finite element model via more detailed material characterization, uniaxial tests will be extended to bending tests to study more compact stowed configurations. The model will be modified and improved to account for the effects of irreversible strains and large strains. Final step for the model will be using the carbon fiber network in the composite as piezoresistive sensing mechanism. Strain sensing together with the developed actuation model can be implemented as a closed loop control for the shape recovery. 


\section{APPENDIX A: FINITE ELEMENT SIMULATIONS}


Case: Sample thermal-electrical-structural simulation

Before developing the actual finite element model, the coupled thermal-electrical-structural solver of ABAQUS has been tried via some simple cases for its performance and accuracy of the modeling. It is also used for checking the extent of parameters derived in multiphysics analysis chapter.

As a simple case, a rectangular block of conductive material has been chosen with dimensions $100 \mathrm{~mm} \times 20 \mathrm{~mm} \times 2 \mathrm{~mm}$. The sample is constrained at the two opposing edges and potential difference is applied. For simplicity, the material is assumed to heat up uniformly. Stresses are measured in the material and compared with analytical solutions.

The model is meshed with 3 elements along thickness direction. Since this sample model is just to validate the analysis and modeling capabilities, values have been assigned as material parameters, therefore, no units will be presented here (Table A.1). Voltage difference of $15 \mathrm{~V}$ has been applied to the opposing edges of the sample. Simulation duration is set to 1 seconds.

Table A.1: Sample model parameters

\begin{tabular}{|c|c|}
\hline Density & $780 \times 10^{-12}$ \\
\hline Modulus & $200 \times 10^{3}$ \\
\hline$v$ & 0.3 \\
\hline Specific heat & $9 \times 10^{8}$ \\
\hline Thermal conductivity & 1 \\
\hline Coefficient of thermal expansion & $1 \times 10^{-3}$ \\
\hline Electrical conductivity & 750 \\
\hline
\end{tabular}

Obtaining an analytical solution for this problem is very straightforward. First, the total amount of heat generated in beam must be found due to Joule heating. Following equations from multiphysics 
analysis chapter,

$$
\begin{gathered}
Q_{\text {gen }}=\frac{V^{2} \times k}{L} \times w \times h \times t, \\
Q_{\text {gen }}=\frac{15^{2} \times 750}{100} \times 20 \times 2 \times 1=67500[\text { units of energy] },
\end{gathered}
$$

where $\mathrm{k}$ is the electrical conductivity of the material. Assuming there is no heat transfer to the surrounding medium, all of the generated heat will cause the sample to heat up. Generated heat will be equal to the product of temperature rise and heat capacity, i.e.

$$
\begin{gathered}
Q_{\text {gen }}=\rho \times w \times L \times h \times C_{p} \times \Delta T, \\
\Delta T=\frac{67500}{780 \times 10^{-12} \times 20 \times 100 \times 2 \times 9 \times 10^{8}}=24.038[K],
\end{gathered}
$$

Finally compressive stress in constrained material due to thermal loading is given by

$$
\begin{gathered}
\sigma=\Delta T \times E \times \alpha, \\
\sigma_{x}=24.038 \times 200 \times 10^{3} \times 1 \times 10^{-3}=-4807.6[\text { units of stress] }
\end{gathered}
$$


and corresponding strains at transverse directions can be found from,

$$
\varepsilon_{x}=\varepsilon_{y}=\frac{-v}{E}\left(\sigma_{x}\right)+\alpha \Delta T=0.03215[]
$$

Comparison of analytical and simulation results can be seen in Table A.2.

Table A.2: Comparison of analytical solution and simulation

\begin{tabular}{|c|c|c|c|}
\hline & Simulation & Analytical & Error \% \\
\hline Stress & -4808 & -4807.6 & $-8.32 \times 10^{-3}$ \\
\hline Current density & 112.5 & 112.5 & 0 \\
\hline Strain in transverse directions & 0.03125 & 0.03215 & 0 \\
\hline$\Delta \mathrm{T}$ & 24.04 & 24.038 & $-8.32 \times 10^{-3}$ \\
\hline
\end{tabular}

The material parameters derived in multiphysics discussion found to be adequate for this case. The coupled thermal-electrical-structural solver is validated and chosen for subsequent finite element models. Caution must be taken when using the actual parameters, and consistent units must be used. This is especially difficult when dealing with multiple domains such as thermal and electrical together with mechanical. It is suggested to use base SI units as property units in the analysis.

Case: Heat flux from one side

This thesis is based on a viscoelastic model for SMPs, with time-temperature superposition principle. Shape recovery is treated as a function of temperature through shift factors. After programming SMP uniaxially, if a heat flux is applied to one surface of the SMP, SMP is expected to bend due to non-symmetric temperature (hence strain) field. This case is tested in finite element model. In the simulation, the SMP layer is programmed uniaxially by high temperature deformation, stress relaxation at constant deformation, cooling at the same deformation, and removal of the constraint. 
After the stowage period, a heat flux is applied to the top surface in the model.

In this simulation only the middle portion of the model is programmed, therefore the shape memory response can only be seen in that region.

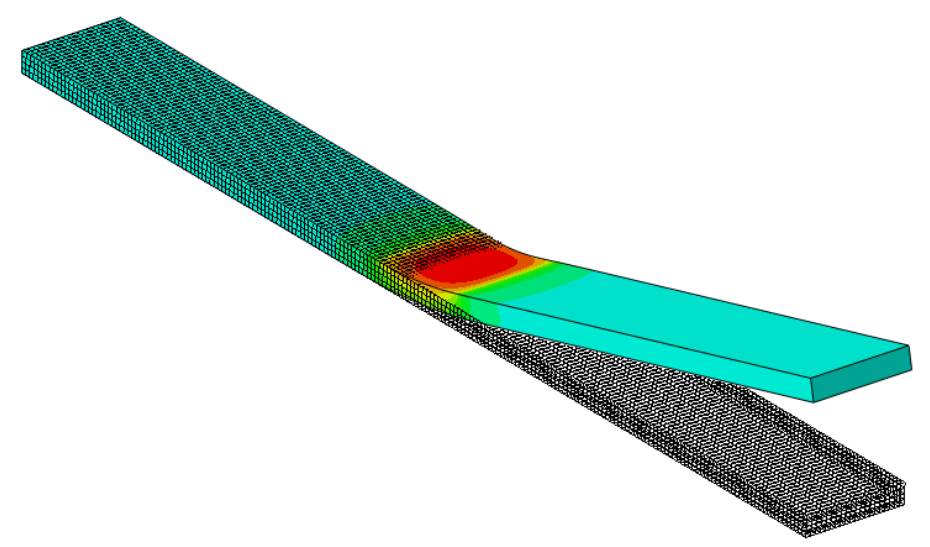

Figure A.1: Temperature field during heating

When heated, there will be a non-uniform temperature field through the thickness. The top surface have higher temperature than the bottom surface, therefore the top surface will try go obtain its original configuration much faster than bottom surface (i.e. contraction at top surface will be much higher), and due to compatibility, material will bend in heated region (Figure A.1).

By controlling the temperature field along the thickness, it is possible to achieve hinge-like behavior from uniaxially programmed SMP. By a careful control of the temperature, it is also possible to fix the shape of the SMP (if it is cooled down at this shape, hinge-like shape will be preserved). 
Case: Bending simulation of CNP/SMP composite

Bending simulations of CNP/SMP composite are carried out, with the same dimensions and properties of the materials as manufactured composite, to assess the performance of the model in bending. For these simulations, rotation boundary condition is specified at the reference point ( $1 \mathrm{rad})$ at one end while the other end is constrained in all directions. Full thermo-mechanical cycle is observed including activation. Figure A.2 shows the shape recovery of the composite over time.

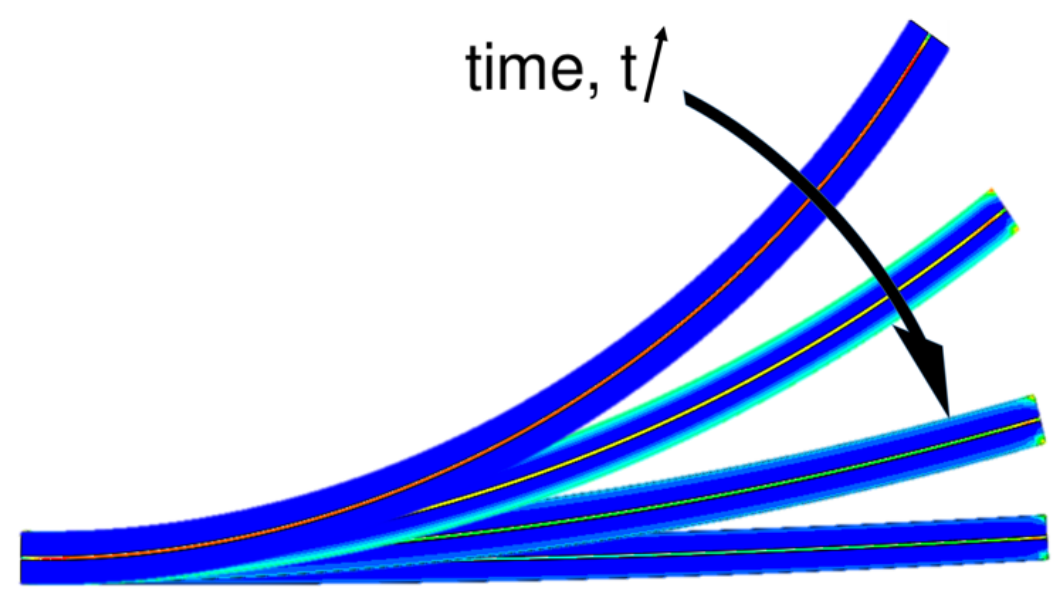

Figure A.2: Shape recovery of the composite in flexure 
APPENDIX B: SENSITIVITY ANALYSIS 
In finite element simulations, some material parameters are assumed. The effect of these parameters on the overall composite behavior needs to be checked via sensitivity analysis. In this section, the dependence of the shape recovery on assumed parameters is analyzed by varying the value of the assumed parameters.

The interfacial thermal conductance value between CNP and SMP was varied several order of magnitudes in simulations. This value becomes important especially during the activation step. Figure B.1 shows the changes in surface temperature of the composite with different interfacial thermal conductance values, while remaining of the parameters are being equal.

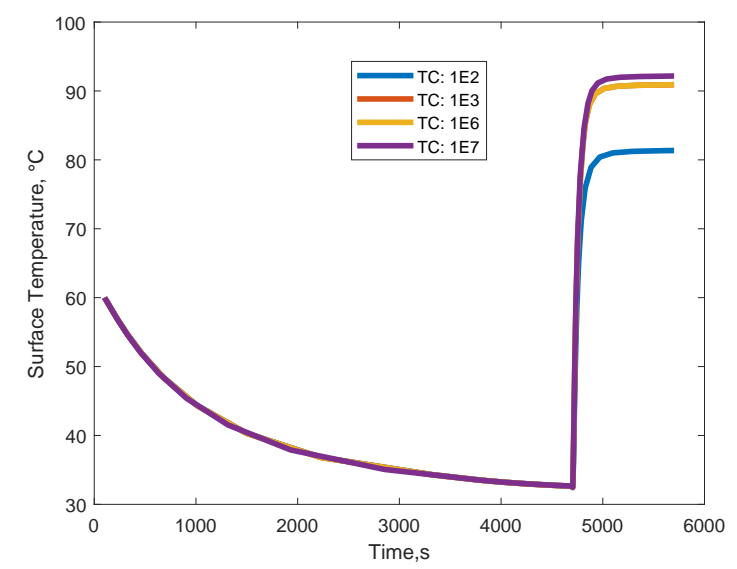

Figure B.1: Effect of interfacial thermal conductance on surface temperature

Figure B.1 shows that although the interfacial thermal conductance value is changed drastically, surface temperature variations are small until the value of interfacial thermal conductance becomes small. High thermal conductance promotes easy diffusion of heat to SMP, causing the temperature of the CNP and inner layer of the SMP to be almost identical. As the thermal conductance value becomes lower, heat transfer is slowed down at the interface, causing the CNP temperature to rise 
considerably compared to the temperature of the inner layer of SMP. However, this high temperature gradient at the interface will promote heat transfer again, as the driving force for heat transfer is temperature gradient. As the thermal conductance at the interface becomes lower, the variation of the surface temperature and therefore shape recovery becomes larger (Figure B.2). For fully isolated interface, surface temperature of the composite will not change as well as the shape of the composite.

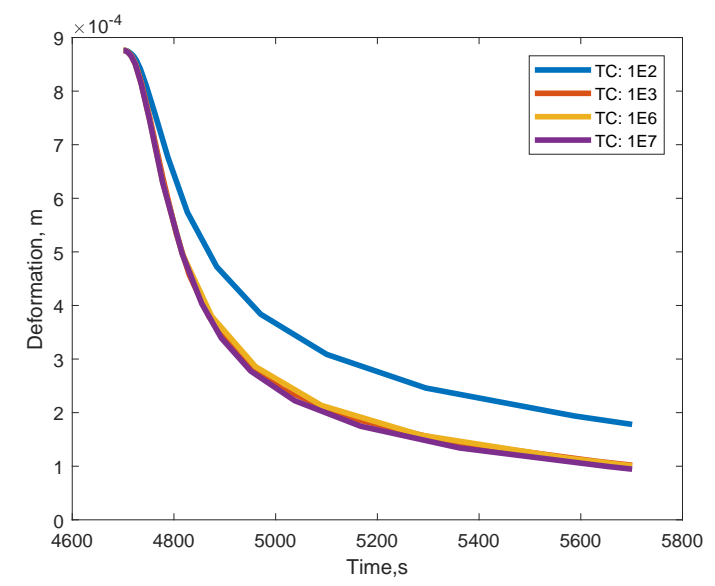

Figure B.2: Effect of interfacial thermal conductance on shape recovery

For viscoelastic materials, the rate of deformation affects the load. For higher deformation rates, the load required for deformation is larger than the cases with slower deformation rates. Figure B.3 show the effect of the deformation rate on the load. 


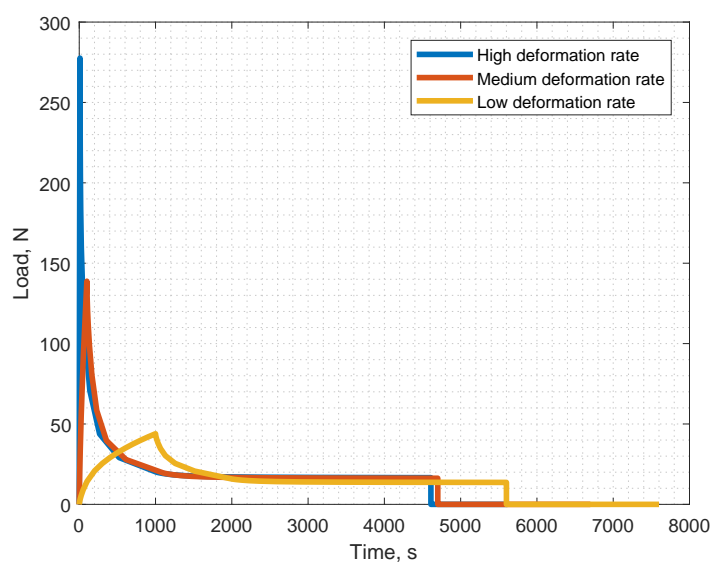

Figure B.3: Effect of deformation rate during programming

Similarly, the duration of high temperature constant deformation duration (during hold step) changes the relaxation behavior. The material relaxes more if more time is allowed during hold step (Figure B.4).

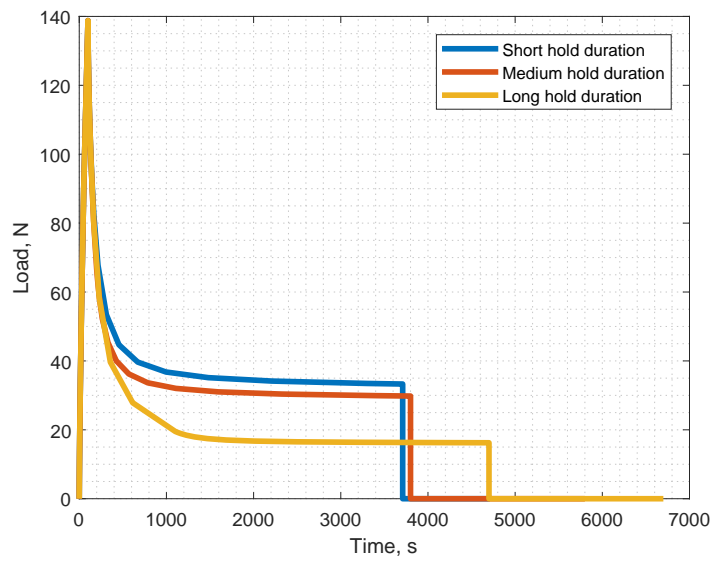

Figure B.4: Effect of hold duration during programming 
The location of electrodes of the power source may influence the electrical potential field in the CNP and may affect the heat generation in that layer. Figure B.5 and Figure B.6 shows simulation results of the electrical potential field of CNP for the electrodes located at half width and quarter width, respectively. It can be seen that, electrodes located at quarter width causes non-uniform potential gradient at the vicinity of the electrodes but this effect becomes very small away from electrode locations.

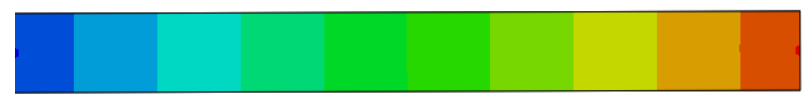

Figure B.5: Effect of location of electrodes on potential field, located at half width

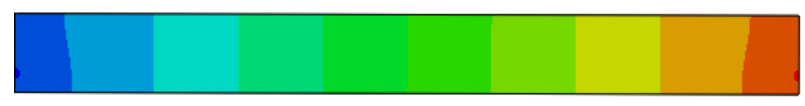

Figure B.6: Effect of location of electrodes on potential field, located at quarter width 


\section{APPENDIX C: SHAPE RECOVERY FORMULATION AND OPTIMIZATION FOR SHAPE CHANGE}


The system of coupled differential equations presented in the Multiphysics Model chapter is implemented in MATLAB code and solved assuming a deformed initial condition in individual branches in the Wiechert model. The solution of the system of differential equation is used to calculate the strain in the equilibrium branch, which is the overall strain of the SMP. Solving for the shape recovery requires the shift factor history. Figure C.1 shows the shape recovery profiles for different uniform shift factors during actuation. The lower the value of the shift factor, the faster the shape recovery will be. Note that, the effect of the coefficient of thermal expansion is not included in the solution, therefore no expansion peaks are observed in the activation period.

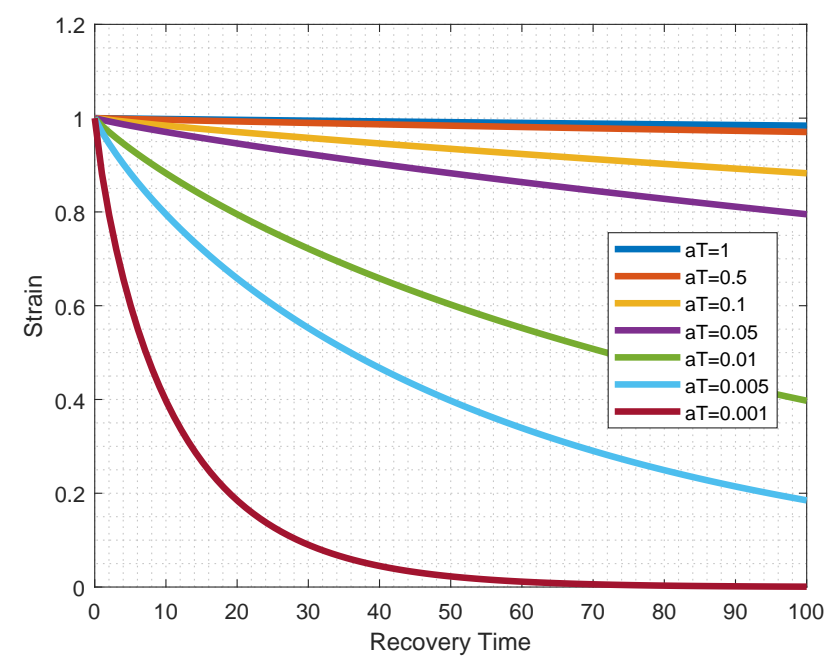

Figure C.1: Shape recovery profile for different shift factors

The solution of the problem can also be utilized to predict the shape recovery when temperature history is given. Assuming the composite has a uniform temperature at all times, shift factors can be found from the temperature history and later be used in the solution to determine shape recovery (Figure C.2 and Figure C.3). 

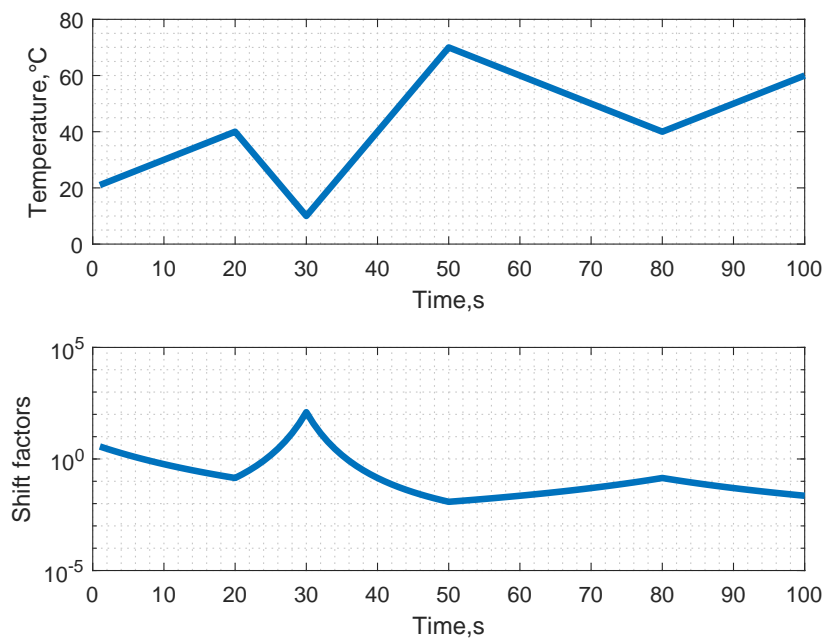

Figure C.2: Temperature and shift factor history

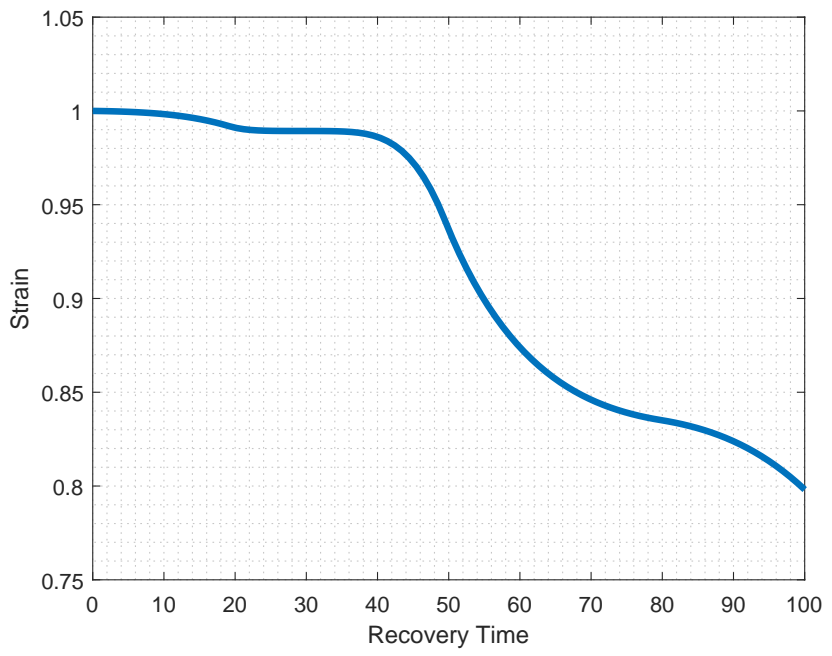

Figure C.3: Shape recovery profile 
If a particular shape recovery profile is needed, the shift factor history can be determined by solving the problem backwards via optimization. Figure C.4 shows the desired shape recovery profile. Using optimization toolbox on MATLAB, the shift factor history can be found to achieve desired shape change. Note that, shift factors can be used to obtain temperature field with respect to time, as well as voltage input over time. Also it is important to note that, the result for this particular optimization is not realistic due to sharp changes of the shift factors, which results in non-smooth temperature history (high temperature variations in very small time steps). The condition for smoothness can be implemented in the optimization to narrow down temperature histories that are physcially achievable.

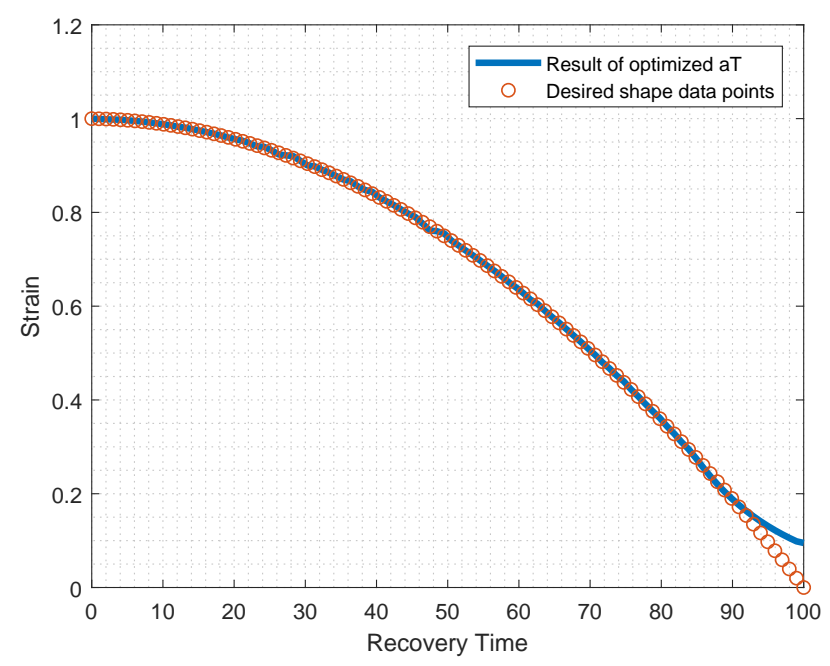

Figure C.4: Shape recovery profile defined by function $\varepsilon=1-\mathrm{t}^{2} / 10000$ 


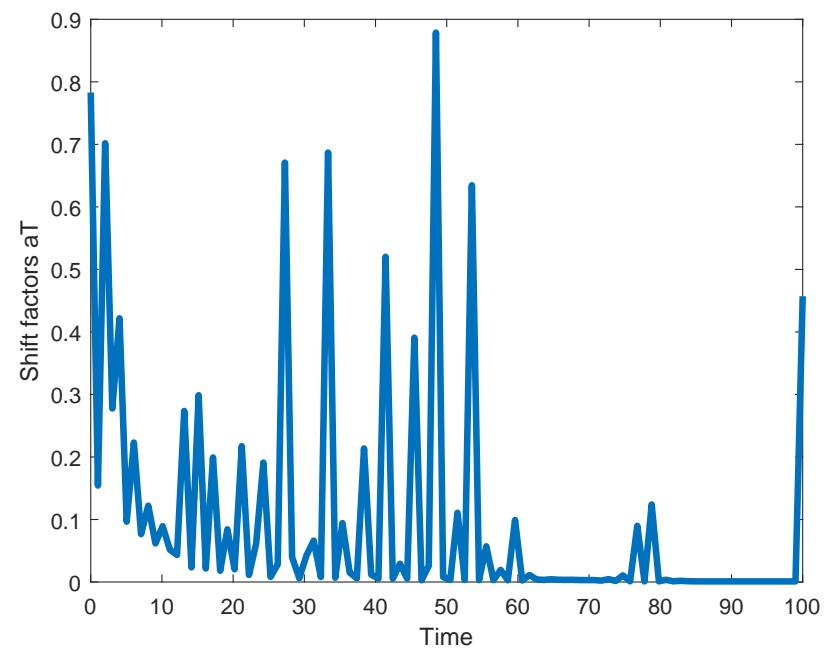

Figure C.5: Shift factor history for profile defined by $\varepsilon=1-\mathrm{t}^{2} / 10000$ 


\section{APPENDIX D: STRESS RELAXATION EXPERIMENTS}


Material linearity or nonlinearity can be determined from the creep compliance or the relaxation modulus at different stress or strain levels. In order to determine linearity, stress relaxation tests are used in this work. Linearity suggests that modulus of the material is independent of the strain level, and only a function of time. After plotting an isochronus strain-stress diagram, if modulus variation is linear, material is said to be linear. From the linearity condition, following equations can be written,

$$
\begin{gathered}
E\left(t=t_{1}\right)=\frac{\sigma_{a}\left(t=t_{1}\right)}{\varepsilon_{0 a}}=\frac{\sigma_{b}\left(t=t_{1}\right)}{\varepsilon_{0 b}}=\frac{\sigma_{c}\left(t=t_{1}\right)}{\varepsilon_{0 c}}, \\
E\left(t=t_{2}\right)=\frac{\sigma_{a}\left(t=t_{2}\right)}{\varepsilon_{0 a}}=\frac{\sigma_{b}\left(t=t_{2}\right)}{\varepsilon_{0 b}}=\frac{\sigma_{c}\left(t=t_{2}\right)}{\varepsilon_{0 c}}, \\
E(t)=\frac{\sigma_{a}(t)}{\varepsilon_{0 a}}=\frac{\sigma_{b}(t)}{\varepsilon_{0 b}}=\frac{\sigma_{c}(t)}{\varepsilon_{0 c}} .
\end{gathered}
$$

Four tests are carried out with different deformation levels and stress relaxation is observed for two minutes. Deformation and load plots are extracted and 3 time values are chosen for comparison of moduli $(t=0 \mathrm{~s}, \mathrm{t}=20 \mathrm{~s}, \mathrm{t}=120 \mathrm{~s})$. Linear fits are employed for these 3 isochronus data. Ideally, stress relaxation curves should fall in between lines of $t=0$ and $t=120 \mathrm{~s}$. Some variation can be seen, especially in the first test (Test1), but the trend can be considered as linear. In Figure D.2 dashed lines are isochronus lines.

Figure D. 3 shows a sample strain field for $\varepsilon_{1}$ for room temperature stress relaxation test. At the boundaries of the area of interest, experimental errors are present, and the strain value is averaged over the area of interest. 


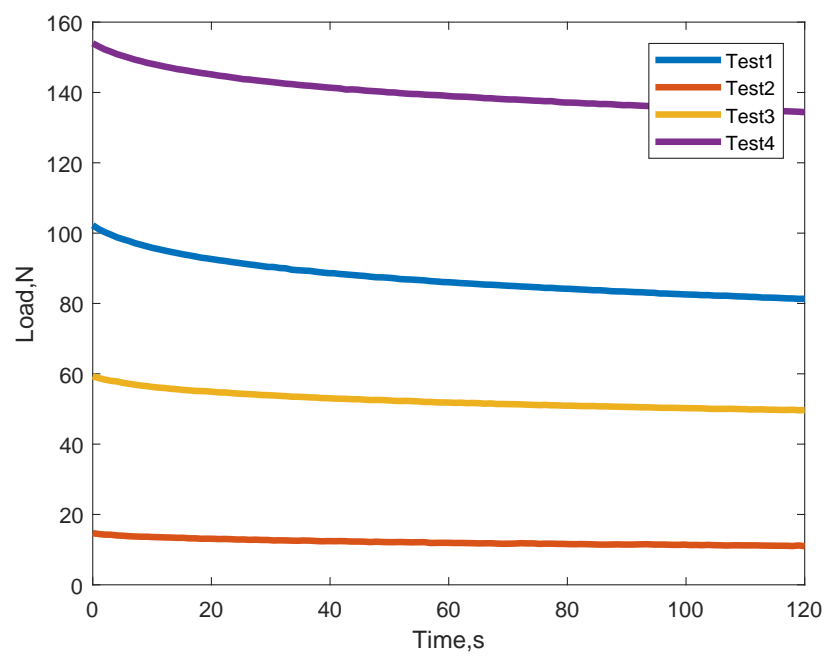

Figure D.1: Relaxation for different deformation levels

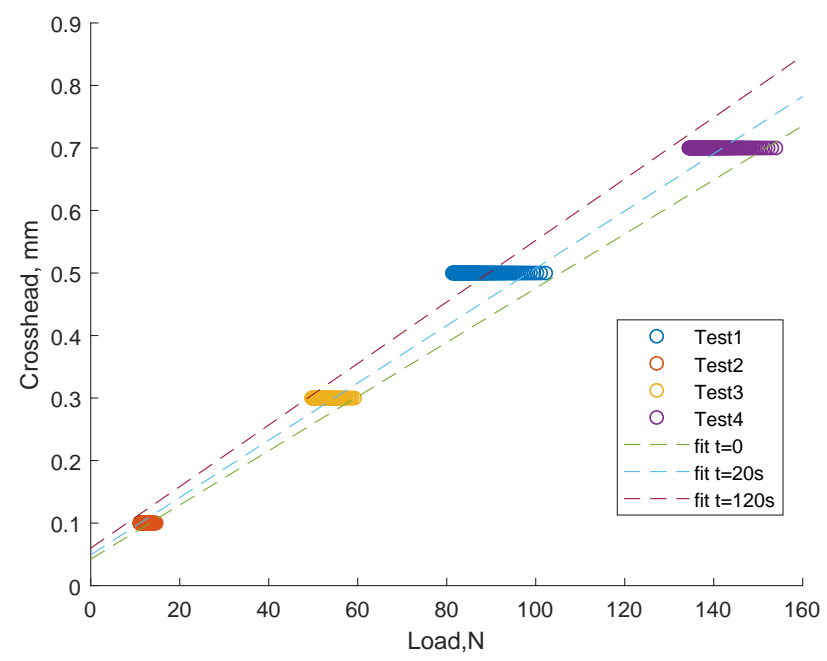

Figure D.2: Isochronus plot 


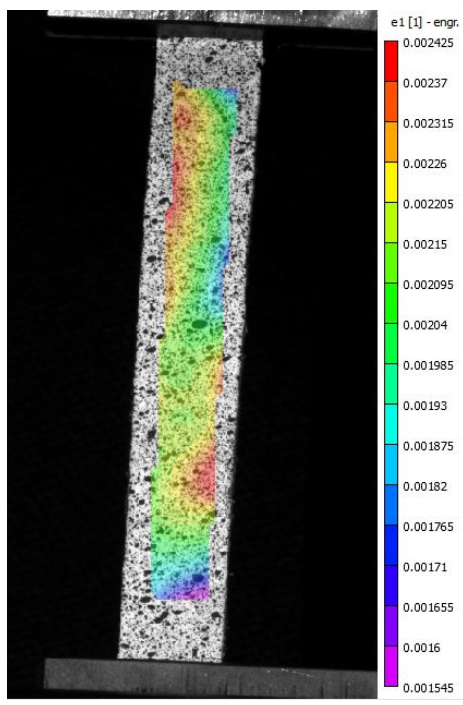

Figure D.3: Sample strain field during stress relaxation test

Figure D.4 shows stress relaxation tests for different temperatures for several samples. Repeatability can be observed among SMP samples with small variations in relaxation curves.

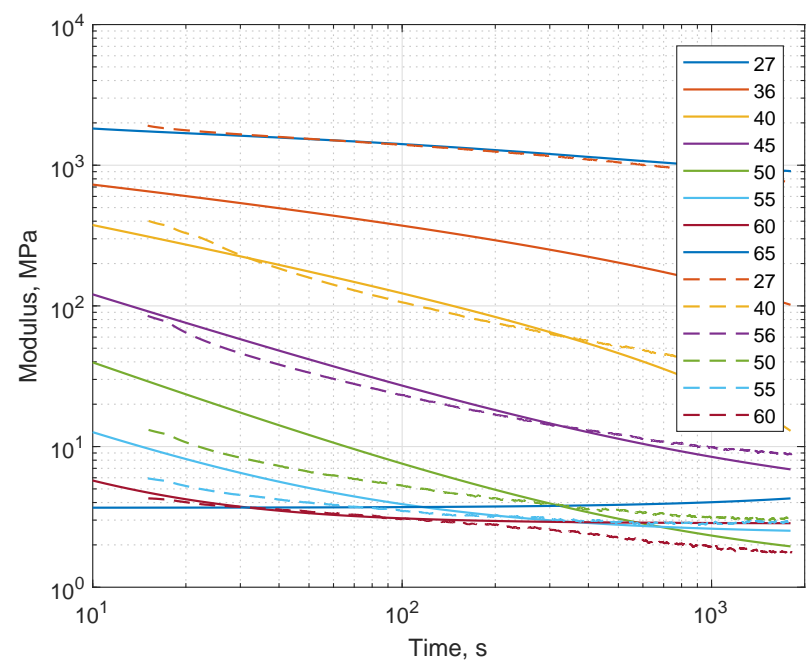

Figure D.4: Stress relaxation tests at different temperatures 
Figure D.5 and Figure D.6 displays the intermediate steps for the formation of master curve of SMP. Stress relaxation tests are shifted horizontally to create a smooth master curve and they are averaged with moving averaging scheme. The averaged master curve is used to fit the Prony series.

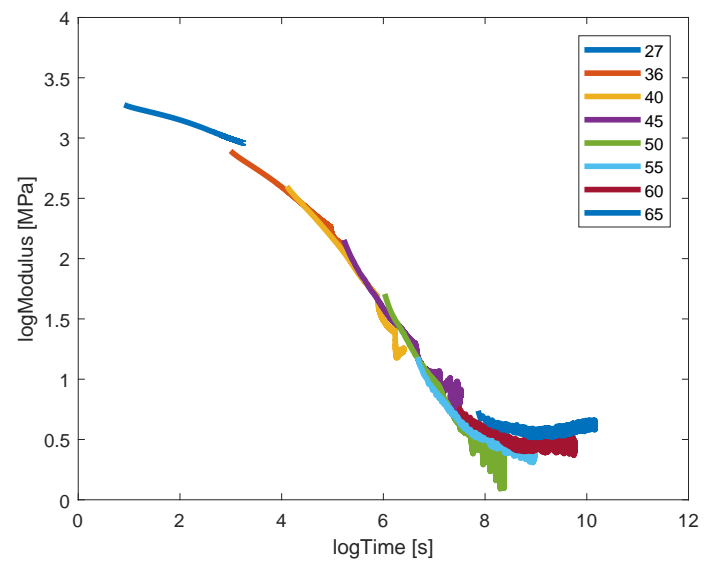

Figure D.5: Shifted stress relaxation tests

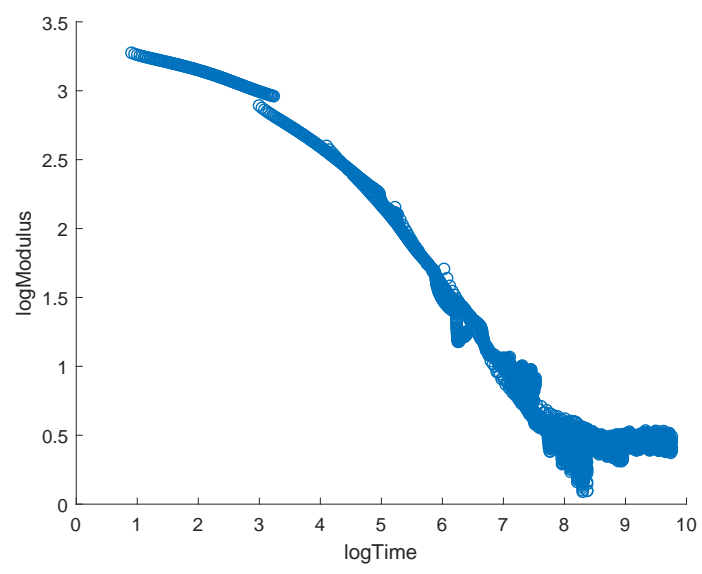

Figure D.6: Sorted master curve 


\section{LIST OF REFERENCES}

[1] X.-J. Han, Z.-Q. Dong, M.-M. Fan, Y. Liu, J.-H. li, Y.-F. Wang, Q.-J. Yuan, B.-J. Li, and S. Zhang, "ph-induced shape-memory polymers," Macromolecular rapid communications, vol. 33, no. 12, pp. 1055-1060, 2012.

[2] W. Guo, C.-H. Lu, R. Orbach, F. Wang, X.-J. Qi, A. Cecconello, D. Seliktar, and I. Willner, "ph-stimulated dna hydrogels exhibiting shape-memory properties," Advanced Materials, vol. 27, no. 1, pp. 73-78, 2015.

[3] J. Mendez, P. K. Annamalai, S. J. Eichhorn, R. Rusli, S. J. Rowan, E. J. Foster, and C. Weder, "Bioinspired mechanically adaptive polymer nanocomposites with water-activated shapememory effect," Macromolecules, vol. 44, no. 17, pp. 6827-6835, 2011.

[4] X. Gu and P. T. Mather, "Water-triggered shape memory of multiblock thermoplastic polyurethanes (tpus)," Rsc Advances, vol. 3, no. 36, pp. 15783-15791, 2013.

[5] H. Du and J. Zhang, "Solvent induced shape recovery of shape memory polymer based on chemically cross-linked poly (vinyl alcohol)," Soft Matter, vol. 6, no. 14, pp. 3370-3376, 2010.

[6] A. Lendlein, H. Jiang, O. Jünger, and R. Langer, "Light-induced shape-memory polymers," Nature, vol. 434, no. 7035, p. 879, 2005.

[7] D. Habault, H. Zhang, and Y. Zhao, "Light-triggered self-healing and shape-memory polymers," Chemical Society Reviews, vol. 42, no. 17, pp. 7244-7256, 2013.

[8] J. Leng, X. Wu, and Y. Liu, "Infrared light-active shape memory polymer filled with nanocarbon particles," Journal of Applied Polymer Science, vol. 114, no. 4, pp. 2455-2460, 2009. 
[9] A. M. Schmidt, "Electromagnetic activation of shape memory polymer networks containing magnetic nanoparticles," Macromolecular Rapid Communications, vol. 27, no. 14, pp. 11681172, 2006.

[10] S. Conti, M. Lenz, and M. Rumpf, "Modeling and simulation of magnetic-shape-memory polymer composites," Journal of the Mechanics and Physics of Solids, vol. 55, no. 7, pp. 1462 $-1486,2007$.

[11] C. Steven, L. Michael, S. Mark, and B. Rory, "Elastic memory composites (emc) for deployable industrial and commercial applications," in Proc. of SPIE, pp. 35-47, 2005.

[12] J. Leng, X. Lan, Y. Liu, and S. Du, "Shape-memory polymers and their composites: stimulus methods and applications," Progress in Materials Science, vol. 56, no. 7, pp. 1077-1135, 2011.

[13] C. M. Yakacki, R. Shandas, C. Lanning, B. Rech, A. Eckstein, and K. Gall, "Unconstrained recovery characterization of shape-memory polymer networks for cardiovascular applications," Biomaterials, vol. 28, no. 14, pp. 2255 - 2263, 2007.

[14] W. Sokolowski, A. Metcalfe, S. Hayashi, L. Yahia, and J. Raymond, "Medical applications of shape memory polymers," Biomedical Materials, vol. 2, pp. S23-S27, mar 2007.

[15] Y. Liu, H. Du, L. Liu, and J. Leng, "Shape memory polymers and their composites in aerospace applications: a review," Smart Materials and Structures, vol. 23, no. 2, p. 023001, 2014.

[16] A. Rakow, K. Hedin, and B. Anthony, "Development of high specific power solar arrays with shape memory polymer hinge lines," in 2018 AIAA Spacecraft Structures Conference, p. 2206, 2018. 
[17] X. Lan, Y. Liu, H. Lv, X. Wang, J. Leng, and S. Du, "Fiber reinforced shape-memory polymer composite and its application in a deployable hinge," Smart Materials and Structures, vol. 18, p. 024002 , jan 2009.

[18] D. Campbell, M. Lake, M. Scherbarth, E. Nelson, and R. Six, Elastic Memory Composite Material: An Enabling Technology for Future Furlable Space Structures.

[19] R. Taylor, E. Abrahamson, R. Barrett, D. Codell, and P. Keller, "Passive deployment of an emc boom using radiant energy in thermal vacuum," 042007.

[20] P. Keller, M. Lake, D. Codell, R. Barrett, R. Taylor, and M. Schultz, Development of Elastic Memory Composite Stiffeners for a Flexible Precision Reflector.

[21] J. S. E. J. Kang, P. R. K., and T. T. L. (2012), “U.s. patent no. us9796159b2. washington, dc: U.s. patent and trademark office.."

[22] R. Barrett, W. Francis, E. Abrahamson, M. Lake, and M. Scherbarth, "Qualification of elastic memory composite hinges for spaceflight applications," in 47th AIAA/ASME/ASCE/AHS/ASC Structures, Structural Dynamics, and Materials Conference 14th AIAA/ASME/AHS Adaptive Structures Conference 7th, p. 2039, 2006.

[23] M. Lake and F. Beavers, The Fundamentals of Designing Deployable Structures with Elastic Memory Composites.

[24] H. Lu and J. Gou, "Study on 3-d high conductive graphene buckypaper for electrical actuation of shape memory polymer," Nanoscience and Nanotechnology Letters, vol. 4, no. 12, pp. 1155-1159, 2012.

[25] H. Lu, F. Liang, Y. Yao, J. Gou, and D. Hui, "Self-assembled multi-layered carbon nanofiber nanopaper for significantly improving electrical actuation of shape memory polymer nanocomposite," Composites Part B: Engineering, vol. 59, pp. 191-195, 2014. 
[26] R. W. Mailen, Y. Liu, M. D. Dickey, M. Zikry, and J. Genzer, "Modelling of shape memory polymer sheets that self-fold in response to localized heating," Soft Matter, vol. 11, no. 39, pp. 7827-7834, 2015.

[27] X. Wang, J. Sparkman, and J. Gou, "Strain sensing of printed carbon nanotube sensors on polyurethane substrate with spray deposition modeling," Composites Communications, vol. 3, pp. 1-6, 2017.

[28] C. Liu, H. Qin, and P. Mather, "Review of progress in shape-memory polymers," Journal of Materials Chemistry - J MATER CHEM, vol. 17, 042007.

[29] C. Schmidt, A. M. S. Chowdhury, K. Neuking, and G. Eggeler, "Studies on the cycling, processing and programming of an industrially applicable shape memory polymer," High Performance Polymers, vol. 23, pp. 300-307, June 2011.

[30] T. D. Nguyen, C. M. Yakacki, P. D. Brahmbhatt, and M. L. Chambers, "Modeling the relaxation mechanisms of amorphous shape memory polymers," Advanced Materials, vol. 22, no. 31, pp. 3411-3423, 2010.

[31] M. Baghani, J. Arghavani, and R. Naghdabadi, "A finite deformation constitutive model for shape memory polymers based on Hencky strain," Mechanics of Materials, vol. 73, pp. 1-10, June 2014.

[32] Y. Liu, K. Gall, M. L. Dunn, A. R. Greenberg, and J. Diani, “Thermomechanics of shape memory polymers: Uniaxial experiments and constitutive modeling," International Journal of Plasticity, vol. 22, no. 2, pp. 279 - 313, 2006.

[33] Y.-C. Chen and D. C. Lagoudas, "A constitutive theory for shape memory polymers. part i: Large deformations," Journal of the Mechanics and Physics of Solids, vol. 56, no. 5, pp. 1752 $-1765,2008$. 
[34] J. R. Lin and L. W. Chen, "Mechanical-viscoelastic model and wlf relationship in shape memorized linear ether-type polyurethanes," Journal of Polymer Research, vol. 6, pp. 35-40, 011999.

[35] J. R. Lin and L. W. Chen, "Shape-memorized crosslinked ester-type polyurethane and its mechanical viscoelastic model," Journal of Applied Polymer Science, vol. 73, no. 7, pp. 13051319, 1999.

[36] K. Yu, Q. Ge, and H. J. Qi, "Reduced time as a unified parameter determining fixity and free recovery of shape memory polymers," Nature communications, vol. 5, p. 3066, 2014.

[37] "Thermomechanical constitutive model of shape memory polymer," Mechanics of Materials, vol. 33 , no. 10 , pp. $545-554,2001$.

[38] H. TOBUSHI, T. HASHIMOTO, S. HAYASHI, and E. YAMADA, "Thermomechanical constitutive modeling in shape memory polymer of polyurethane series," Journal of Intelligent Material Systems and Structures - J INTEL MAT SYST STRUCT, vol. 8, pp. 711-718, 08 1997.

[39] J. Lai and A. Bakker, "3-D schapery representation for non-linear viscoelasticity and finite element implementation," p. 10.

[40] M. Behl and A. Lendlein, "Shape-memory polymers," Materials Today, vol. 10, pp. 20-28, Apr. 2007.

[41] M. Baghani, R. Naghdabadi, and J. Arghavani, "A large deformation framework for shape memory polymers: Constitutive modeling and finite element implementation," Journal of Intelligent Material Systems and Structures, vol. 24, pp. 21-32, Jan. 2013. 
[42] F. Castro, K. K. Westbrook, J. Hermiller, D. U. Ahn, Y. Ding, and H. J. Qi, "Time and Temperature Dependent Recovery of Epoxy-Based Shape Memory Polymers," Journal of Engineering Materials and Technology, vol. 133, no. 2, p. 021025, 2011.

[43] Y. Liu, H. Du, L. Liu, and J. Leng, "Shape memory polymers and their composites in aerospace applications: a review," Smart Materials and Structures, vol. 23, p. 023001, Feb. 2014.

[44] "Theoretical studies on microbuckling mode of elastic memory composites," Acta Mechanica Solida Sinica, vol. 23, no. 1, pp. 20 - 28, 2010.

[45] F. L. Jimenez and S. Pellegrino, "Folding of thin-walled composite structures with a soft matrix," AIAA Paper, no. 2009-2633, pp. 1-18, 2009.

[46] F. L. JimÃ Šnez and S. Pellegrino, "Failure of carbon fibers at a crease in a fiber-reinforced silicone sheet," Journal of Applied Mechanics, vol. 80, no. 1, p. 011020, 2013.

[47] F. L. Jiménez and S. Pellegrino, "Folding of fiber composites with a hyperelastic matrix," International Journal of Solids and Structures, vol. 49, no. 3-4, pp. 395-407, 2012.

[48] D. Bergman and B. Yang, "A finite element model of shape memory polymer composite beams for space applications," International Journal for Numerical Methods in Engineering, vol. 103, no. 9, pp. 671-702, 2015.

[49] H. Meng and G. Li, "A review of stimuli-responsive shape memory polymer composites," Polymer, vol. 54, pp. 2199-2221, Apr. 2013.

[50] F. Liang, R. Sivilli, J. Gou, Y. Xu, and B. Mabbott, "Electrical actuation and shape recovery control of shape-memory polymer nanocomposites," International Journal of Smart and Nano Materials, vol. 4, pp. 167-178, Sept. 2013. 
[51] X. Luo and P. T. Mather, "Conductive shape memory nanocomposites for high speed electrical actuation," Soft Matter, vol. 6, no. 10, pp. 2146-2149, 2010.

[52] Y. Liu, H. Lv, X. Lan, J. Leng, and S. Du, "Review of electro-active shape-memory polymer composite," Composites Science and Technology, vol. 69, no. 13, pp. 2064-2068, 2009.

[53] M. Raja, S. H. Ryu, and A. Shanmugharaj, "Thermal, mechanical and electroactive shape memory properties of polyurethane (pu)/poly (lactic acid)(pla)/cnt nanocomposites," European Polymer Journal, vol. 49, no. 11, pp. 3492-3500, 2013.

[54] H. Lu, W. M. Huang, and J. Leng, "Functionally graded and self-assembled carbon nanofiber and boron nitride in nanopaper for electrical actuation of shape memory nanocomposites," Composites Part B: Engineering, vol. 62, pp. 1-4, 2014.

[55] I. S. Gunes, G. A. Jimenez, and S. C. Jana, "Carbonaceous fillers for shape memory actuation of polyurethane composites by resistive heating," Carbon, vol. 47, no. 4, pp. 981-997, 2009.

[56] H. Lu, Y. Liu, and J. Leng, "Carbon nanopaper enabled shape memory polymer composites for electrical actuation and multifunctionalization," Macromolecular Materials and Engineering, vol. 297, no. 12, pp. 1138-1147, 2012.

[57] J. Leng, W. Huang, X. Lan, Y. Liu, and S. Du, "Significantly reducing electrical resistivity by forming conductive ni chains in a polyurethane shape-memory polymer/carbon-black composite," Applied Physics Letters, vol. 92, no. 20, p. 204101, 2008.

[58] H. Lu, Y. Liu, J. Gou, J. Leng, and S. Du, "Synergistic effect of carbon nanofiber and carbon nanopaper on shape memory polymer composite," Applied Physics Letters, vol. 96, no. 8, p. $084102,2010$. 
[59] Y. Kuronuma, T. Takeda, Y. Shindo, F. Narita, and Z. Wei, "Electrical resistance-based strain sensing in carbon nanotube/polymer composites under tension: Analytical modeling and experiments," Composites Science and Technology, vol. 72, pp. 1678-1682, Sept. 2012.

[60] Z. Wang and X. Ye, "A numerical investigation on piezoresistive behaviour of carbon nanotube/polymer composites: mechanism and optimizing principle," Nanotechnology, vol. 24, p. 265704, July 2013.

[61] A. Oskouyi, U. Sundararaj, and P. Mertiny, "Current-voltage characteristics of nanoplateletbased conductive nanocomposites," Nanoscale research letters, vol. 9, p. 369, 072014.

[62] Y. Zare and K. Y. Rhee, "A simple methodology to predict the tunneling conductivity of polymer/CNT nanocomposites by the roles of tunneling distance, interphase and CNT waviness," RSC Advances, vol. 7, no. 55, pp. 34912-34921, 2017.

[63] Y. Wang and X. Zhao, "A theoretical model of effective electrical conductivity and piezoresistivity of carbon nanotube composites," Philosophical Magazine Letters, vol. 98, pp. 38-43, Jan. 2018.

[64] A. Oskouyi and P. Mertiny, "Monte carlo model for the study of percolation thresholds in composites filled with circular conductive nano-disks," vol. 10, pp. 403-408, 122011.

[65] S. Rawal, J. Brantley, and N. Karabudak, "Development of carbon nanotube-based composite for spacecraft components," in 2013 6th International Conference on Recent Advances in Space Technologies (RAST), (Istanbul, Turkey), pp. 13-19, IEEE, June 2013.

[66] P. E. Lopes, F. van Hattum, C. M. Pereira, P. J. Nóvoa, S. Forero, F. Hepp, and L. Pambaguian, "High CNT content composites with CNT Buckypaper and epoxy resin matrix: Impregnation behaviour composite production and characterization," Composite Structures, vol. 92, pp. 1291-1298, May 2010. 
[67] F. Deng and Q.-S. Zheng, "An analytical model of effective electrical conductivity of carbon nanotube composites," Applied Physics Letters, vol. 92, no. 7, p. 071902, 2008.

[68] Z. Han and A. Fina, "Thermal conductivity of carbon nanotubes and their polymer nanocomposites: a review," Progress in polymer science, vol. 36, no. 7, pp. 914-944, 2011.

[69] F. Deng and Q. Zheng, "Interaction models for effective thermal and electric conductivities of carbon nanotube composites," Acta Mechanica Solida Sinica, vol. 22, no. 1, pp. 1-17, 2009.

[70] K. Yu, A. J. McClung, G. P. Tandon, J. W. Baur, and H. J. Qi, “A thermomechanical constitutive model for an epoxy based shape memory polymer and its parameter identifications," Mechanics of Time-Dependent Materials, vol. 18, no. 2, pp. 453-474, 2014.

[71] H. F. Brinson and L. C. Brinson, "Polymer engineering science and viscoelasticity," An Introduction, 2008.

[72] www.pyrografproducts.com, "Pyrograf $囚-$-iii."

[73] J. Wen, "Heat capacities of polymers," in Physical properties of polymers handbook, pp. 145154, Springer, 2007.

[74] B. Wunderlich, Thermal analysis of polymeric materials. Springer Science \& Business Media, 2005.

[75] E. Turi, Thermal characterization of polymeric materials. Elsevier, 2012.

[76] T. Osswald and J. P. Hernández-Ortiz, "Polymer processing," Modeling and Simulation. Munich: Hanser, pp. 1-651, 2006. 